Japan. J. Math.

Vol. 15, No. 2, 1989

\title{
Singular hyperbolic systems, VII. Asymptotic analysis for Fuchsian hyperbolic equations in Gevrey classes (2)
}

\author{
By Hidetoshi Tahara
}

(Received February 29, 1988)

In the previous paper [5], the irregularity index $\sigma(\geqq 1)$ was defined for some Fuchsian hyperbolic operators $P$, and the equation $P u=f$ was investigated in the Gevrey class $\mathscr{E}^{\mathscr{s}\}}$ under the condition $1<s<\sigma /(\sigma-1)$. The following is a typical example:

$$
\begin{gathered}
L=\left(t \partial_{t}\right)^{2}-\sum_{i=1}^{n} t^{2 \kappa_{i}} \partial_{x_{i}}^{2}+a(t, x)\left(t \partial_{t}\right)+\sum_{i=1}^{n} t^{l_{i}} b_{i}(t, x) \partial_{x_{i}}+c(t, x), \\
\sigma_{L}=\max \left\{1, \frac{2 \kappa_{1}-l_{1}}{\kappa_{1}}, \cdots, \frac{2 \kappa_{n}-l_{n}}{\kappa_{n}}\right\}
\end{gathered}
$$

(where $(t, x) \in[0, T] \times \boldsymbol{R}^{n}, \kappa_{i}>0(1 \leqq i \leqq n)$ and $l_{i}>0(1 \leqq i \leqq n)$ ).

The purpose of this paper is to study the following case:

$$
s=\sigma /(\sigma-1) .
$$

Roughly speaking, the result established in this paper is as follows: if we treat the equation $P u=f$ in the projective Gevrey class $\mathscr{E}^{\mathscr{( s}}$ (not in the classical one $\mathscr{E}^{[s\}}$ ), we can obtain the same results as in [5] also in the case

$$
s=\sigma /(\sigma-1) \text { and } \Delta_{P} \cap S_{P}=\varnothing
$$

(see Theorem 2).

The reason why we must introduce the class $\mathscr{E}^{(s)}$ is illustrated as follows, Let $A=\left(t \partial_{t}+1\right)\left(t \partial_{t}+2\right)-t^{2 k} \partial_{x}^{2}+t^{l} a \partial_{x}$ (where $(t, x) \in[0, T] \times R, k, l \in\{1,2, \cdots\}$, $l \geqq 2, a \in C$ and $a \neq 0$ ) and recall that the irregularity index $\sigma$ of $A$ is given by $\sigma=\max \{1,(2 k-l) / k\}$. Then, we can see the following:

(i) When $1<s<\sigma /(\sigma-1), A u=f$ is well-posed in $\mathscr{E}^{\{s\}}$ (by [5]).

(ii) When $s=\sigma /(\sigma-1), A u=f$ is not well-posed in $\mathscr{E}^{\{s\}}$ even in the local sense (by an application of [1]).

(iii) When $s=\sigma /(\sigma-1), A u=f$ is well-posed in $\mathscr{E}^{(s)}$ (by Theorem 2).

The condition $\Delta_{P} \cap S_{P}=\varnothing$ is introduced as a necessary and sufficient condition for some formal power series to converge under $s=\sigma /(\sigma-1)$ (see 
Proposition 1). Most of our operators satisfy $\Delta_{P} \cap S_{P}=\varnothing$; in particular, if $P$ is of the second order, $\Delta_{P} \cap S_{P}=\varnothing$ is trivially satisfied. But, there are examples of the case $\Delta_{P} \cap S_{P} \neq \varnothing$ and it seems impossible to apply our argument to the case

$$
s=\sigma /(\sigma-1) \text { and } \Delta_{P} \cap S_{P} \neq \varnothing
$$

(by Proposition 1). Hence, it is still an open problem to decide whether the condition $\Delta_{P} \cap S_{P}=\varnothing$ (under $s=\sigma /(\sigma-1)$ ) is essential or not.

\section{§1. Preliminaries}

First, let us explain the operator $P$ treated here, the irregularity index $\sigma(\geqq 1)$ of $P$, the function spaces and the problem.

The operator treated here is as follows:

$$
P=\left(t \partial_{t}\right)^{m}+\sum_{\substack{j+1 \alpha \mid \leqq m \\ j<m}} t^{l(j, \alpha)} a_{j, \alpha}(t, x)\left(t \partial_{t}\right)^{j} \partial_{x}^{\alpha}
$$

where $(t, x)=\left(t, x_{1}, \cdots, x_{n}\right) \in[0, T] \times \boldsymbol{R}^{n}(T>0), \partial_{t}=\partial / \partial t, \partial_{x}=\left(\partial / \partial x_{1}, \cdots, \partial / \partial x_{n}\right)$, $m \in N(=\{1,2,3, \cdots\}), \alpha=\left(\alpha_{1}, \cdots, \alpha_{n}\right) \in Z_{+}^{n}\left(=\{0,1,2, \cdots\}^{n}\right),|\alpha|=\alpha_{1}+\cdots+\alpha_{n}$ and $\partial_{x}^{\alpha}=\left(\partial / \partial x_{1}\right)^{\alpha_{1}} \cdots\left(\partial / \partial x_{n}\right)^{\alpha_{n}}$. On the coefficients, we assume that $a_{j, \alpha}(t, x) \in$ $C^{0}\left([0, T] \times \boldsymbol{R}^{n}\right) \cap C^{\infty}\left((0, T) \times \boldsymbol{R}^{n}\right)(j+|\alpha| \leqq m$ and $j<m)$ and that they satisfy $\left(t \partial_{t}\right)^{i} \partial_{x}^{\beta} a_{j, \alpha}(t, x) \in C^{0}\left([0, T] \times \boldsymbol{R}^{n}\right)$ for any $(i, \beta) \in Z_{+} \times \boldsymbol{Z}_{+}^{n}$. As to the hyperbolicity, we assume the following conditions $\left(\mathrm{A}_{\kappa}\right)$ and $(\mathrm{B})$ :

$\left(\mathrm{A}_{\kappa}\right) \quad l(j, \alpha) \in \boldsymbol{R}(j+|\alpha| \leqq m$ and $j<m)$ satisfy

$$
\begin{cases}l(j, \alpha)=\kappa_{1} \alpha_{1}+\cdots+\kappa_{n} \alpha_{n}, & \text { when } j+|\alpha|=m \text { and } j<m, \\ l(j, \alpha)>0, & \text { when } j+|\alpha|<m \text { and }|\alpha|>0, \\ l(j, \alpha) \geqq 0, & \text { when } j+|\alpha|<m \text { and }|\alpha|=0\end{cases}
$$

for some $\kappa=\left(\kappa_{1}, \cdots, \kappa_{n}\right) \in \boldsymbol{R}^{n}$ such that $\kappa_{i}>0(1 \leqq i \leqq n)$.

(B) All the roots $\lambda_{i}(t, x, \xi)(1 \leqq i \leqq m)$ of the equation (in $\left.\lambda\right)$

$$
\lambda^{m}+\sum_{\substack{j+|\alpha|=m \\ j<m}} a_{j, \alpha}(t, x) \lambda^{j} \xi^{\alpha}=0
$$

are real, simple and bounded on $\left\{(t, x, \xi) \in[0, T] \times \boldsymbol{R}^{n} \times \boldsymbol{R}^{n} ;|\xi|=1\right\}$.

Then, $P$ is the Fuchsian hyperbolic operator discussed in [5]. Recall that the characteristic exponents $\rho_{1}(x), \cdots, \rho_{m}(x)$ of $P$ are defined by the roots of

$$
\rho^{m}+\sum_{j<m} a_{j}(x) \rho^{j}=0
$$

where $a_{j}(x)=\left.\left[t^{l(j,(0, \cdots, 0))} a_{j,(0, \ldots, 0)}(t, x)\right]\right|_{t=0}(j<m)$. Also, recall that the irreg- 
ularity index $\sigma(\geqq 1)$ of $P$ is defined by

$$
\sigma=\max \left[1, \max _{\substack{j+|\alpha|<m \\|\alpha|>0}}\left\{\min _{\tau \in \mathfrak{S}_{n}}\left(\max _{1 \leqq r \leqq n} M_{j, \alpha}(\tau, r)\right)\right\}\right],
$$

where $\mathbb{S}_{n}$ is the permutation group of $n$-numbers and

$$
M_{j, \alpha}(\tau, r)=\frac{\sum_{i=1}^{r}\left(\kappa_{\tau(i)}-\kappa_{\tau(r)}\right) \alpha_{z(i)}+(m-j) \kappa_{\tau(r)}-l(j, \alpha)}{(m-j-|\alpha|) \kappa_{\tau(r)}} .
$$

In [5], we discussed the equation $P u=f$ in $C^{\infty}\left([0, T], \mathscr{E}^{[s\}}\left(\boldsymbol{R}^{n}\right)\right)$ or $C^{\infty}((0, T)$, $\left.\mathscr{E}^{\{s\}}\left(\boldsymbol{R}^{n}\right)\right)$ under the condition $1<s<\sigma /(\sigma-1)$. But, in this paper, we will consider the equation $P u=f$ in $C^{\infty}\left([0, T], \mathscr{E}^{(s)}\left(\boldsymbol{R}^{n}\right)\right)$ or $C^{\infty}\left((0, T), \mathscr{E}^{(s)}\left(\boldsymbol{R}^{n}\right)\right)$ under the condition $1<s \leqq \sigma /(\sigma-1)$.

Here, $\mathscr{E}^{(s)}\left(\boldsymbol{R}^{n}\right)$ [resp. $\mathscr{E}^{[s\}}\left(\boldsymbol{R}^{n}\right)$ ] denotes the set of all the functions $f(x) \in$ $C^{\infty}\left(\boldsymbol{R}^{n}\right)$ satisfying the following: for any compact subset $K$ of $\boldsymbol{R}^{n}$ and for any $h>0$, there is a $C>0$ [resp. for any compact subset $K$ of $\boldsymbol{R}^{n}$, there are $h>0$ and $C>0$ ] such that

$$
\sup _{x \in K}\left|\partial_{x}^{\alpha} f(x)\right| \leqq C h^{|\alpha|}(|\alpha| !)^{s} \quad \text { for any } \alpha \in Z_{+}^{n} .
$$

When $s=\infty$, we put $\mathscr{E}^{(\infty)}\left(\boldsymbol{R}^{n}\right)=\mathscr{E}\left(\boldsymbol{R}^{n}\right)$. Note that $\mathscr{E}^{(s)}\left(\boldsymbol{R}^{n}\right) \subseteq \mathscr{E}^{\{s\}}\left(\boldsymbol{R}^{n}\right)$ holds for $1<s<\infty$. For simplicity, we often write $\mathscr{E}^{*}$ instead of $\mathscr{E}^{\mathscr{E}\}}$ or $\mathscr{E}^{\mathscr{E}(s)}$; that is, $\mathscr{E}^{*}$ with $*=\{s\}$ means $\mathscr{E}^{\mathscr{f} s}$, and $\mathscr{E}^{*}$ with $*=(s)$ means $\mathscr{E}^{(s)}$. By $C^{\infty}\left([0, T], \mathscr{E}^{*}\left(\boldsymbol{R}^{n}\right)\right)$ [resp. $\left.C^{\infty}\left((0, T), \mathscr{E}^{*}\left(\boldsymbol{R}^{n}\right)\right)\right]$ we denote the set of all infinitely differentiable functions on $[0, T]$ [resp. $(0, T)]$ with values in $\mathscr{E}^{*}$ equipped with the usual topology.

Then, by following the discussion in [5] we can obtain the following result.

Theorem 1. Let $P$ be the operator in (1.1) satisfying $\left(\mathrm{A}_{k}\right)$ and $(\mathrm{B})$. Assume the following conditions:

(1) $1<s<\sigma /(\sigma-1)$.

(2) $*=\{s\}$ or $(s)$.

( 3 ) $t^{l(j, \alpha)} a_{j, \alpha}(t, x) \in C^{\infty}\left([0, T], \mathscr{E}^{*}\left(\boldsymbol{R}^{n}\right)\right)(j+|\alpha| \leqq m$ and $j<m)$.

Then we have the following results (I) and (II).

( I ) (Unique solvability). If $\rho_{i}(x) \notin \boldsymbol{Z}_{+}$holds for any $x \in \boldsymbol{R}^{n}$ and $1 \leqq i \leqq m$, the equation

$$
P u=f \quad \text { in } C^{\infty}\left([0, T], \mathscr{E} *\left(\boldsymbol{R}^{n}\right)\right)
$$

is uniquely solvable.

(II) (Asymptotic expansions). If $\rho_{i}(x)-\rho_{j}(x) \notin Z$ holds for any $x \in \boldsymbol{R}^{n}$ 
and $1 \leqq i \neq j \leqq m$, the general solution of the equation

$$
P u=0 \quad \text { in } C^{\infty}\left((0, T), \mathscr{E} *\left(\boldsymbol{R}^{n}\right)\right)
$$

is characterized as follows. (II- 1$)$ Any solution $u(t, x)(=u) \in C^{\infty}\left((0, T), \mathscr{E} *\left(\boldsymbol{R}^{n}\right)\right)$ of (1.5) is expanded asymptotically into the form

$$
u(t, x) \sim \sum_{i=1}^{m}\left\{\varphi_{i}(x) t^{\rho_{i}(x)}+\sum_{k=1}^{\infty} \sum_{h=0}^{m k} \varphi_{i, k, h}(x) t^{\rho_{i}(x)+k}(\log t)^{h}\right\}
$$

(as $t \rightarrow+0$ ) for some unique $\varphi_{i}(x), \varphi_{i, k, h}(x) \in \mathscr{E}^{*}\left(\boldsymbol{R}^{n}\right)(1 \leqq i \leqq m, 1 \leqq k<\infty$ and $0 \leqq h \leqq m k)$. (II-2) Conversely, for any $\varphi_{1}(x), \cdots, \varphi_{m}(x) \in \mathscr{E}^{*}\left(\boldsymbol{R}^{n}\right)$ there exist a unique solution $u(t, x)(=u) \in C^{\infty}\left((0, T), \mathscr{E} *\left(\boldsymbol{R}^{n}\right)\right)$ of $(1.5)$ and unique coefficients $\varphi_{i, k, h}(x) \in \mathscr{E}^{*}\left(\boldsymbol{R}^{n}\right)(1 \leqq i \leqq m, 1 \leqq k<\infty$ and $0 \leqq h \leqq m k)$ such that the asymptotic relation in (II-1) holds.

In fact, the case $*=\{s\}$ was already proved in [5] and the case $*=(s)$ may be proved by an argument quite parallel to the case $*=\{s\}$ (see also $\S \S 4$ and 5 ).

Hence, our target is only the case $s=\sigma /(\sigma-1)$. Since it seems impossible to have good results in the case $*=\{s\}$ with $s=\sigma /(\sigma-1)$ (as is seen in the introduction), we will discuss only the case $*=(s)$ with $s=\sigma /(\sigma-1)$ from now on.

\section{§. Main theorem}

Now, let us consider the equation $P u=f$ in $C^{\infty}\left([0, T], \mathscr{E}^{(s)}\left(\boldsymbol{R}^{n}\right)\right)$ or $C^{\infty}\left((0, T), \mathscr{E}^{(s)}\left(\boldsymbol{R}^{n}\right)\right)$ under the condition $s=\sigma /(\sigma-1)$.

Let $P$ be the operator in (1.1) satisfying $\left(\mathrm{A}_{k}\right)$ and (B). Put

$$
\mathscr{J}=\left\{(j, \alpha) \in \boldsymbol{Z}_{+} \times \boldsymbol{Z}_{+}^{n} ; j+|\alpha|<m \text { and }|\alpha|>0\right\},
$$

let $\sigma(\geqq 1)$ be as in (1.2), and let $M_{j, \alpha}(\tau, r)$ be as in (1.3). Define $\sigma_{j, \alpha}(\geqq 1)$ by

$$
\sigma_{j, \alpha}=\max \left[1, \min _{\tau \in \mathbb{S}_{n}}\left(\max _{1 \leqq r \leqq n} M_{j, \alpha}(\tau, r)\right)\right]
$$

Then, we have $\sigma=\max \left\{\sigma_{j, \alpha} ;(j, \alpha) \in \mathscr{J}\right\}$. Put $\Delta_{P}$ as follows:

$$
\Delta_{P}=\left\{(j, \alpha) \in \mathscr{J} ; \sigma_{j, \alpha}=\sigma\right\} .
$$

Let $\kappa=\left(\kappa_{1}, \cdots, \kappa_{n}\right) \in \boldsymbol{R}^{n}$ be the one in $\left(\mathrm{A}_{k}\right)$. For $\alpha=\left(\alpha_{1}, \cdots, \alpha_{n}\right) \in \boldsymbol{Z}_{+}^{n}$, denote by $S_{\kappa}(\alpha)$ the set of all $l \in \boldsymbol{R}$ satisfying the following (i) and (ii): (i) $0<l<\langle\kappa, \alpha\rangle$, and (ii) there are $\tau \in \widetilde{S}_{n}$ and $p \in\{1, \cdots, n-1\}$ such that

$$
\left\{\begin{array}{l}
l=\kappa_{\tau(1)} \alpha_{\tau(1)}+\cdots+\kappa_{\tau(p)} \alpha_{z(p)}, \\
\left\{\kappa_{\tau(1)}, \cdots, \kappa_{\tau(p)}\right\}<\left\{\kappa_{\tau(p+1)}, \cdots, \kappa_{\tau(n)}\right\} .
\end{array}\right.
$$

Here, $\langle\kappa, \alpha\rangle=\kappa_{1} \alpha_{1}+\cdots+\kappa_{n} \alpha_{n}$, and $\left\{a_{1}, \cdots, a_{p}\right\}<\left\{b_{1}, \cdots, b_{q}\right\}$ means that $a_{i}<b_{j}$ 
holds for any $i$ and $j$. Put $S_{P}$ as follows:

$$
S_{P}=\left\{(j, \alpha) \in \mathscr{J} ; l(j, \alpha) \in S_{\kappa}(\alpha)\right\} .
$$

Then, the main theorem of this paper is stated as follows.

Theorem 2. Let $P$ be the operator in (1.1) satisfying $\left(\mathrm{A}_{\kappa}\right)$ and $(\mathrm{B})$. Assume the following conditions:

$$
\begin{aligned}
& \text { (1) } s=\sigma /(\sigma-1) \quad \text { and } \Delta_{P} \cap S_{P}=\varnothing . \\
& \text { (2) } *=(s) \text {. } \\
& \text { (3) } t^{l(j, \alpha)} a_{j, \alpha}(t, x) \in C^{\infty}\left([0, T], \mathscr{E}^{(s)}\left(\boldsymbol{R}^{n}\right)\right)(j+|\alpha| \leqq m \text { and } j<m) \text {. }
\end{aligned}
$$

Then, the same results as in Theorem 1:-(I) (Unique solvability) and (II) (Asymptotic expansions)-are valid.

In the case $\sigma=1$ (i.e. $s=\infty$ ), Theorem 2 was already proved in [3, 4]. In the case $\sigma>1$ (i.e. $1<s<\infty$ ), Theorem 2 will be proved in $\S \S 4$ and 5 .

As to the condition $\Delta_{P} \cap S_{P}=\varnothing$, we remark the following.

REMARK. (1) When $\sigma=1, l(j, \alpha) \geqq\langle\kappa, \alpha\rangle$ holds for any $(j, \alpha) \in \mathscr{J}$; therefore in this case the condition $\Delta_{P} \cap S_{P}=\varnothing$ is trivially satisfied.

(2) When $\kappa_{1}=\cdots=\kappa_{n}, S_{\kappa}(\alpha)=\varnothing$ holds for any $\alpha \in Z_{+}^{n}$; therefore in this case the condition $\Delta_{P} \cap S_{P}=\varnothing$ is trivially satisfied.

(3) When $|\alpha|=1, S_{\kappa}(\alpha)=\varnothing$ holds for any $\kappa=\left(\kappa_{1}, \cdots, \kappa_{n}\right)$; therefore in the case $m=2$ the condition $\Delta_{P} \cap S_{P}=\varnothing$ is trivially satisfied.

(4) When $m=3, n=2$ and $0<\kappa_{1}<\kappa_{2}, S_{\kappa}((1,0))=S_{\kappa}((0,1))=S_{\kappa}((2,0))=$ $S_{\kappa}((0,2))=\varnothing$ and $S_{k}((1,1))=\left\{\kappa_{1}\right\}$ hold; therefore in this case the condition $\Delta_{P} \cap S_{P}=\varnothing$ is equivalent to the following: $\sigma \neq 2$ or $l(0,(1,1)) \neq \kappa_{1}$.

(5) Put $\Sigma_{m}=\{k / h ; k, h \in Z$ and $1 \leqq h<k \leqq m-1\}$. Then we can see the following: when $\sigma \notin \Sigma_{m}$, we have $\Delta_{P} \cap S_{P}=\varnothing$. Note that for any $k / h \in \Sigma_{m}$ we can find an example such that $\sigma=k / h$ and $\Delta_{P} \cap S_{P} \neq \varnothing$.

By Theorems 1 and 2, we have obtained good results in the following two cases:

$$
\begin{aligned}
& \text { (C-1) } 1<s<\sigma /(\sigma-1) . \\
& \text { (C-2) } s=\sigma /(\sigma-1) \text { and } \Delta_{P} \cap S_{P}=\varnothing .
\end{aligned}
$$

But, our method employed here cannot be applied to the case

$$
\text { (C-3) } s=\sigma /(\sigma-1) \text { and } \Delta_{P} \cap S_{P} \neq \varnothing ;
$$

therefore, it is still an open problem to study the case (C-3).

In $\S \S 4$ and 5 [resp. in [5]], we will prove Theorem 2 [resp. we proved 
Theorem 1] by reducing the problem to the one of the convergence of a majorant series $M_{a}\left(\left\{\beta\left(J_{k}\right)\right\}, C, R, t\right)$ which appears naturally in the estimation of our formal solution (based on the method of successive approximations). In our proof, the following fact is essential: the majorant series $M_{a}\left(\left\{\beta\left(J_{k}\right)\right\}, C, R, t\right)$ converges, if and only if (C-1) or (C-2) holds.

Precisely, it is stated as follows. Let $\mathscr{J}$ be as in (2.1), and put $\mathscr{J}^{1}=\mathscr{J}$, $\mathscr{J}^{2}=\mathscr{J} \times \mathscr{J}, \mathscr{J}^{3}=\mathscr{J} \times \mathscr{J} \times \mathscr{J}, \cdots$. For $J_{k}=\left(\left(j_{1}, \alpha_{(1)}\right), \cdots,\left(j_{k}, \alpha_{(k)}\right)\right) \in \mathscr{J}^{k}$, we denote by $\mathscr{M}\left(J_{k}\right)$ the set of all $\left(\beta_{(1)}, \cdots, \beta_{(k)}\right) \in \boldsymbol{Z}_{+}^{n} \times \cdots \times \boldsymbol{Z}_{+}^{n}$ satisfying the following:

$$
\left\{\begin{array}{l}
(0, \cdots, 0) \leqq \beta_{(i)} \leqq \alpha_{(i)}(i=1, \cdots, k), \\
\left\langle\kappa, \beta_{(1)}+\cdots+\beta_{(i)}\right\rangle \leqq l\left(j_{1}, \alpha_{(1)}\right)+\cdots+l\left(j_{i}, \alpha_{(i)}\right) \quad(i=1, \cdots, k) .
\end{array}\right.
$$

Let $a>0$. For $J_{k}=\left(\left(j_{1}, \alpha_{(1)}\right), \cdots,\left(j_{k}, \alpha_{(k)}\right)\right) \in \mathscr{J}^{k}, \beta\left(J_{k}\right)=\left(\beta_{(1)}, \cdots, \beta_{(k)}\right) \in \mathscr{M}\left(J_{k}\right)$, $C>0$ and $R>0$, we put

$$
\begin{aligned}
& F_{a}\left(J_{k}, \beta\left(J_{k}\right), C, R, t\right) \\
&= C^{k+1} t^{l_{1}+\cdots+l_{k}-\left\langle x, \beta_{(1)}+\cdots+\beta_{(k)}\right\rangle} \times R^{\left(\left|\alpha_{(1)}\right|-\left|\beta_{(1)}\right|\right)+\cdots+\left(\left|\alpha_{(k)}\right|-\left|\beta_{(k)}\right|\right)} \\
& \times \frac{1}{a_{k+1}} \times \frac{\left[\left(\left|\alpha_{(1)}\right|-\left|\beta_{(1)}\right|\right)+\cdots+\left(\left|\alpha_{(k)}\right|-\left|\beta_{(k)}\right|\right)\right] !^{s}}{\left(a_{1}\right)^{m-j_{1}-\left|\beta_{(1)}\right|} \times \cdots \times\left(a_{k}\right)^{m-j_{k}-\left|\beta_{(k)}\right|}}
\end{aligned}
$$

where $l_{i}=l\left(j_{i}, \alpha_{(i)}\right)(i=1, \cdots, k), a_{1}=a$ and

$$
a_{i+1}=a+l_{1}+\cdots+l_{i}-\left\langle\kappa, \beta_{(1)}+\cdots+\beta_{(i)}\right\rangle \quad(i=1, \cdots, k) .
$$

Then, the majorant series $M_{a}\left(\left\{\beta\left(J_{k}\right)\right\}, C, R, t\right)$ which appears in $\S 4$ is given by

$$
M_{a}\left(\left\{\beta\left(J_{k}\right)\right\}, C, R, t\right)=\sum_{k=1}^{\infty} \sum_{J_{k} \in g^{k}} F_{a}\left(J_{k}, \beta\left(J_{k}\right), C, R, t\right)
$$

(see (4.7)). As to the convergence of $M_{a}\left(\left\{\beta\left(J_{k}\right)\right\}, C, R, t\right)$, we have the following result (the proof will be given in $\S 6$ ).

Proposition 1. Let $1<s<\infty, a>0, C>0$ and $T>0$. Then, we have the following results (I) and (II).

(I) The following three conditions (I-1), (I-2) and (I-3) are equivalent to each other.

(I-1) (C-1) or (C-2) holds.

(I-2) There are $R_{0}>0$ and $\beta\left(J_{k}\right) \in \mathscr{M}\left(J_{k}\right)\left(J_{k} \in \mathscr{J}^{k}, k=1,2, \cdots\right)$ such that

$$
M_{a}\left(\left\{\beta\left(J_{k}\right)\right\}, C, R_{0}, T\right)<+\infty .
$$

(I-3) There are $R_{0}>0, T_{0}>0$ and $\beta\left(J_{k}\right) \in \mathscr{M}\left(J_{k}\right)\left(J_{k} \in \mathscr{J}^{k}, k=1,2, \cdots\right)$ such that

$$
M_{a}\left(\left\{\beta\left(J_{k}\right)\right\}, C, R_{0}, T_{0}\right)<+\infty .
$$


(II) The following three conditions (II-1), (II-2) and (II-3) are equivalent to each other.

(II-1) (C-1) holds.

(II-2) For any $R>0$, there are $\beta\left(J_{k}\right) \in \mathscr{M}\left(J_{k}\right)\left(J_{k} \in \mathscr{J}^{k}, k=1,2, \cdots\right)$ such that

$$
M_{a}\left(\left\{\beta\left(J_{k}\right)\right\}, C, R, T\right)<+\infty .
$$

(II-3) For any $R>0$, there are $T_{0}>0$ and $\beta\left(J_{k}\right) \in \mathscr{M}\left(J_{k}\right)\left(J_{k} \in \mathscr{J}^{k}, k=1,2\right.$, ..) such that

$$
M_{a}\left(\left\{\beta\left(J_{k}\right)\right\}, C, R, T_{0}\right)<+\infty .
$$

In the proof of Theorem 1 (in [5]), we used the condition (II-2) in Proposition 1. In the proof of Theorem 2 (in $\S \S 4$ and 5), we will use the condition (I-2) in Proposition 1. This is the reason why our method cannot be applied to the case (C-3).

\section{$\S 3 . \quad$ Some lemmas}

Before the proof of Theorem 2, let us recall here formal norms introduced in $[5, \S 5]$ (see also [2]), and let us present some preparatory lemmas.

Let $p, l \in \boldsymbol{Z}_{+}, r \in \boldsymbol{R}$ and $\kappa=\left(\kappa_{1}, \cdots, \kappa_{n}\right) \in \boldsymbol{R}^{n}$ such that $\kappa_{i}>0(1 \leqq i \leqq n)$. For $f(t, x) \in C^{\infty}\left((0, T), H^{\infty}\left(\boldsymbol{R}^{n}\right)\right)$ and $a(t, x) \in C^{\infty}\left((0, T) \times \boldsymbol{R}^{n}\right)$, we write

$$
\begin{aligned}
\left\|\nabla_{r}^{p, \infty} f(t)\right\| & =\sum_{q=0}^{\infty} \sum_{j+|\alpha| \leqq p} \frac{1}{j ! \alpha !} \sum_{|\beta|=q}\left\|\left(t \partial_{t}+r\right)^{j} \partial_{x}^{\alpha+\beta} f(t)\right\|_{L_{2}\left(\boldsymbol{R}^{n}\right)} \frac{\rho^{q}}{q !}, \\
\left\|\nabla_{r}^{p, \infty} \nabla_{k, r}^{l} f(t)\right\| & =\sum_{j+|\alpha| \leqq l}\left\|\nabla_{r}^{p, \infty} t^{\langle\kappa, \alpha\rangle}\left(t \partial_{t}+r\right)^{j} \partial_{x}^{\alpha} f(t)\right\|, \\
\left\|\nabla^{p, \infty} a\right\|_{\infty} & =\sum_{q=0}^{\infty} \sum_{j+|\alpha| \leqq p} \frac{1}{j ! \alpha !} \sum_{|\beta|=q}\left\|\left(t \partial_{t}\right)^{j} \partial_{x}^{\alpha+\beta} a\right\|_{L^{\infty}(\Omega)} \frac{\rho^{q}}{q !}
\end{aligned}
$$

(where $\langle\kappa, \alpha\rangle=\kappa_{1} \alpha_{1}+\cdots+\kappa_{n} \alpha_{n}$ and $\Omega=(0, T) \times \boldsymbol{R}^{n}$ ). Also, for a differential operator $R$ of the form

$$
R=\sum_{j+|\alpha| \leqq m} t^{\langle\kappa, \alpha\rangle} a_{j, \alpha}(t, x)\left(t \partial_{t}\right)^{j} \partial_{x}^{\alpha}
$$

we write

$$
\left\|\nabla^{p, \infty} R\right\|_{\infty}=\sum_{j+|\alpha| \leqq m}\left\|\nabla^{p, \infty} a_{j, \alpha}\right\|_{\infty} .
$$

For basic properties of these formal norms, see $[5, \S 5]$.

Let $1 \leqq s<\infty$ and put

$$
\theta_{s}(\rho)=\sum_{q=0}^{\infty}(q !)^{s} \frac{\rho^{q}}{q !} .
$$


For a formal power series $\varphi(t, \rho)$ in $\rho$, we write

$$
\varphi(t, \rho) \in \mathscr{E}^{(s)} \text { uniformly on }[0, T],
$$

if $\varphi(t, \rho)$ satisfies the following: for any $\varepsilon>0$ there is a $C_{\varepsilon}>0$ such that $\varphi(t, \rho)$ $\ll C_{\varepsilon} \theta_{s}(\varepsilon \rho)$ holds on $[0, T]$. Here, $\sum_{q=0}^{\infty} a_{q} \rho^{q} \ll \sum_{q=0}^{\infty} b_{q} \rho^{q}$ means that $\left|a_{q}\right| \leqq b_{q}$ holds for any $q$. Then, by Sobolev's lemma we can see

Lemma 1. Let $f(t, x) \in C^{0}\left([0, T], H^{\infty}\left(\boldsymbol{R}^{n}\right)\right)$. Assume that $\operatorname{supp}(f) \subset[0, T]$ $\times K$ holds for some compact subset $K$ of $\boldsymbol{R}^{n}$. Then, the following conditions (i) and (ii) are equivalent:

( i ) $f(t, x) \in C^{0}\left([0, T], \mathscr{E}^{(s)}\left(\boldsymbol{R}^{n}\right)\right)$.

(ii ) $\left\|\nabla_{r}^{0, \infty} f(t)\right\| \in \mathscr{E}^{(s)}$ uniformly on $[0, T]$.

For a positive-valued function $C(\varepsilon)$ in $\varepsilon>0$, we write

$$
\theta_{(s)}(\rho ; C(\varepsilon))=\inf _{\varepsilon>0}\left(C(\varepsilon) \theta_{s}(\varepsilon \rho)\right)
$$

Then, (3.1) is equivalent to the condition that $\varphi(t, \rho) \ll \theta_{(s)}(\rho ; C(\varepsilon))$ holds on $[0, T]$ for some $C(\varepsilon)$. Note that by putting $A=C(1)$ and $C_{0}(\varepsilon)=C(\varepsilon) / A$ we have $\theta_{(s)}(\rho ; C(\varepsilon))=A \theta_{(s)}\left(\rho ; C_{0}(\varepsilon)\right)$ and $C_{0}(1)=1$.

We say that $\varphi(t, \rho)=\sum_{q=0}^{\infty} \varphi_{q}(t) \rho^{q}$ satisfies $\left(\mathrm{M}_{(s)}\right)$ with respect to $C(\varepsilon)$, if $\varphi(t, \rho)$ satisfies the following condition:

$$
\left(\mathrm{M}_{(s)}\right) \quad\left(\inf _{\varepsilon>0}\left(C(\varepsilon) \varepsilon^{p}\right)\right) \frac{(p !)^{s}}{p !} \varphi_{q}(t) \leqq \varphi_{p+q}(t) \quad \text { on }(0, T) \text { for any } p, q \in Z_{+} .
$$

Then, by the same argument as in [5, Lemma 5] we have

Lemma 2. Let $1 \leqq s<\infty$. Assume that $\varphi(t, \rho)(\gg 0)$ satisfies $\left(\mathrm{M}_{(s)}\right)$ with respect to $C(\varepsilon)$. Then, for any $0<2 k \leqq h$ we have

$$
\theta_{(s)}(k \rho ; C(\varepsilon)) \varphi(t, h \rho) \ll 2 \varphi(t, h \rho) .
$$

The following lemma guarrantees that the arguments in [5] with $\theta_{s}(\rho)$ replaced by $\theta_{(s)}(\rho ; C(\varepsilon))$ are also valid.

Lemma 3. Let $1<s<\infty$, let $\varphi(t, \rho)(\gg 0)$ be a formal power series in $\rho$ such that $\varphi(t, \rho) \in \mathscr{E}^{(s)}$ uniformly on $[0, T]$, and let $C_{0}(\varepsilon)$ be a positive-valued function in $\varepsilon>0$ such that $C_{0}(1)=1$. Then, we can find a positive-valued function $C(\varepsilon)$ in $\varepsilon>0$ which satisfies the following conditions (i) and (ii):

( i ) $\varphi(t, \rho) \ll \theta_{(s)}(\rho ; C(\varepsilon))$ holds on $[0, T]$.

(ii) $\theta_{(s)}(\rho ; C(\varepsilon))$ satisfies $\left(\mathrm{M}_{(s)}\right)$ with respect to $C_{0}(\varepsilon)$.

Proof. First, let us make clear the meaning of $\theta_{(s)}(\rho ; C(\varepsilon))$. Denote by 
$\mathscr{F}$ the set of all functions $f(z) \in C^{2}([0, \infty))$ satisfying the following: $f(z)=0$ on $[0,1],(d / d z) f(z) \leqq 0$ on $[0, \infty),(d / d z) f(z) \rightarrow-\infty($ as $z \rightarrow+\infty)$ and $(d / d z)^{2} f(z) \leqq 0$ on $[0, \infty)$. For $f(z) \in \mathscr{F}$, we write

$$
\theta_{s, f}(\rho)=\sum_{q=0}^{\infty} e^{f(q)}(q !)^{s} \frac{\rho^{q}}{q !} .
$$

Then, by comparing the convex sets of the following two types

$$
\begin{aligned}
& \bigcap_{\varepsilon>0}\left\{(z, y) \in R^{2} ; z \geqq 0 \text { and } y \leqq(\log \varepsilon) z+\log C(\varepsilon)\right\}, \\
& \left\{(z, y) \in R^{2} ; z \geqq 0 \text { and } y \leqq f(z)\right\},
\end{aligned}
$$

we can see the following facts (F-1) and (F-2).

(F-1) For any positive-valued function $C(\varepsilon)$ in $\varepsilon>0$ satisfying $C(1)=1$, we can find an $f(z) \in \mathscr{F}$ such that $\theta_{(s)}(\rho ; C(\varepsilon)) \ll \theta_{s, f}(\rho)$.

$(\mathrm{F}-2)$ For any $f(z) \in \mathscr{F}$, we can find a positive-valued function $C(\varepsilon)$ in $\varepsilon>0$ such that $C(1)=1$ and $\theta_{s, f}(\rho)=\theta_{(s)}(\rho ; C(\varepsilon))$.

Now, let us show Lemma 3. Let $\varphi(t, \rho)(\gg 0)$ and $C_{0}(\varepsilon)$ be as in Lemma 3. Choose a positive-valued function $C_{1}(\varepsilon)$ in $\varepsilon>0$ such that $C_{1}(1)=1$ and

$$
\varphi(t, \rho) \ll A \theta_{(s)}\left(\rho ; C_{1}(\varepsilon)\right) \quad \text { on }[0, T]
$$

for some $A>0$. Since $C_{i}(\varepsilon)(i=0,1)$ satisfy $C_{i}(1)=1$, by $(\mathrm{F}-1)$ we can find an $f(z) \in \mathscr{F}$ such that

$$
\theta_{(s)}\left(\rho ; C_{i}(\varepsilon)\right) \ll \theta_{s, f}(\rho) \quad(i=0,1) .
$$

Therefore, to have Lemma 3 it is sufficient to construct a function $g(z) \in \mathscr{F}$ satisfying the following (g-1) and (g-2):

$$
\begin{array}{ll}
\text { (g-1) } & f(z) \leqq g(z) \quad \text { on }[0, \infty) . \\
\text { (g-2) } & (d / d z)^{2}(g(z)+(s-1) \Gamma(1+z)) \geqq 0 \quad \text { on }[0, \infty)
\end{array}
$$

(where $\Gamma(z)$ denotes the gamma-function of Euler). In fact, if such a function $g(z) \in \mathscr{F}$ is obtained, by (3.2), (3.3) and (g-1) we have

$$
\varphi(t, \rho) \ll A \theta_{s, f}(\rho) \ll A \theta_{s, g}(\rho) \quad \text { on }[0, T],
$$

and by (3.3), (g-1) and (g-2) we have

$$
\begin{aligned}
\left.\inf _{\varepsilon>0}\left(C_{0}(\varepsilon) \varepsilon^{p}\right)\right)\left(\frac{(p !)^{s}}{p !}\right)\left(e^{g(q)} \frac{(q !)^{s}}{q !}\right) & \leqq\left(e^{f(p)} \frac{(p !)^{s}}{p !}\right)\left(e^{g(q)} \frac{(q !)^{s}}{q !}\right) \\
& \leqq\left(e^{g(p)} \frac{(p !)^{s}}{p !}\right)\left(e^{g(q)} \frac{(q !)^{s}}{q !}\right) \\
& \leqq e^{g(p+q)} \frac{[(p+q) !]^{s}}{(p+q) !}
\end{aligned}
$$


(for any $\left.p, q \in \boldsymbol{Z}_{+}\right)$which means that $\theta_{s, g}(\rho)$ satisfies $\left(\mathrm{M}_{(s)}\right)$ with respect to $C_{0}(\varepsilon)$; hence, by choosing $C(\varepsilon)$ so that $A \theta_{s, g}(\rho)=\theta_{(s)}(\rho ; C(\varepsilon))$ (by $(\mathrm{F}-2)$ ) we can obtain Lemma 3.

Here, we note the following lemma (the proof will be given later).

Lemma 4. Let $a(z) \in C^{0}([0, \infty))$ be such that $a(z) \geqq 0$ on $[0, \infty)$ and $\int_{0}^{z} a(y) d y \rightarrow \infty($ as $z \rightarrow \infty)$, and let $h(z) \in C^{0}([0, \infty))$ be such that $h(z) \geqq 0$ on $[0, \infty), h(z)$ is increasing in $z$ and $h(z) \rightarrow \infty($ as $z \rightarrow \infty)$. Then, we can find a $b(z) \in C^{0}([0, \infty))$ which satisfies the following conditions: $(b-1) b(z)=0$ on $[0,1],(\mathrm{b}-2) 0 \leqq b(z) \leqq a(z)$ on $[0, \infty),(\mathrm{b}-3) \int_{0}^{z} b(y) d y \rightarrow \infty \quad(a s z \rightarrow \infty)$, and (b-4) $\int_{0}^{z} b(y) d y \leqq h(z)$ on $[0, \infty)$.

By using this lemma, let us construct a function $g(z) \in \mathscr{F}$ satisfying (g-1) and (g-2). Put

$$
\begin{aligned}
& a(z)=(s-1)\left(\frac{d}{d z}\right)^{2} \Gamma(1+z), \\
& h(z)=(-1)\left(\frac{d}{d z}\right) f(z) .
\end{aligned}
$$

Then, $a(z)$ and $h(z)$ satisfy the conditions in Lemma 4. Therefore, by Lemma 4 we have a $b(z) \in C^{0}([0, \infty))$ which satisfies $(b-1) \sim(b-4)$. Hence, by putting

$$
g(z)=(-1) \int_{0}^{z} \int_{0}^{y} b(\tau) d \tau d y
$$

we can obtain a $g(z) \in \mathscr{F}$ satisfying (g-1) and (g-2). Note that the fact $g(z) \in \mathscr{F}$ follows from $(\mathrm{b}-1) \sim(\mathrm{b}-3)$, that $(\mathrm{g}-1)$ follows from $(\mathrm{b}-4)$, and that $(\mathrm{g}-2)$ follows from (b-2).

Q.E.D.

Proof of Lemma 4. When there is a $p \geqq 1$ such that $\int_{p}^{z} a(y) d y \leqq h(z)$ for any $z>p$, by modifying the function

$$
b_{0}(z)= \begin{cases}0, & \text { for } 0 \leqq z \leqq p \\ a(z), & \text { for } z>p\end{cases}
$$

we can obtain a function $b(z)$ in Lemma 4 .

When for any $p \geqq 1$ there is a $q>p$ such that $\int_{p}^{q} a(y) d y>h(q)$, we can choose a sequence $p_{1}, q_{1}, p_{2}, q_{2}, \cdots$ successively so that the following conditions are satisfied: $p_{1} \geqq 1, h\left(p_{1}\right) \geqq 1, q_{k}>p_{k}$,

$$
\sum_{i=1}^{k-1} \int_{p_{i}}^{q_{i}} a(y) d y+\int_{p_{k}}^{z} a(y) d y \begin{cases}<h(z), & \text { for } p_{k} \leqq z<q_{k}, \\ =h(z), & \text { for } z=q_{k},\end{cases}
$$


$p_{k+1}>q_{k}+1$,

$$
\sum_{i=1}^{k} \int_{p_{i}}^{q_{i}} a(y) d y+1 \leqq h\left(p_{k+1}\right)
$$

$(k=1,2, \cdots)$. Therefore, in this case, by modifying the function

$$
b_{0}(z)=\left\{\begin{array}{lll}
0, & \text { for } q_{k-1} \leqq z \leqq p_{k} & (k=1,2, \cdots), \\
a(z), & \text { for } p_{k}<z<q_{k} & (k=1,2, \cdots)
\end{array}\right.
$$

(where $q_{0}=0$ ) we can obtain a function $b(z)$ in Lemma 4.

Q.E.D.

\section{$\S 4$. Proof of (I): Unique solvability}

To have the part (I) in Theorems 1 and 2 , it is sufficient to establish the $\mathscr{E}^{(s)}$-version of [5, Proposition 4] (see also [5, $\S \S 3$ and 7]).

Let $P$ be the operator in (1.1), and let us consider

$$
P\left(t^{r} v\right)=t^{r} g
$$

where $v=v(t, x), g=g(t, x)$, and $r \in R$ is a parameter. As in [5, Proposition 4], we impose here the following conditions:

$\left(B^{\prime}\right)(B)$ is satisfied. In addition, there is a $c>0$ such that

$$
\left|\lambda_{i}(t, x, \xi)-\lambda_{j}(t, x, \xi)\right| \geqq c
$$

holds on $\left\{(t, x, \xi) \in[0, T] \times \boldsymbol{R}^{n} \times \boldsymbol{R}^{n} ;|\xi|=1\right\}$ for any $1 \leqq i \neq j \leqq m$.

$$
\left(\mathrm{D}_{p}^{(s)}\right) \quad\left\|\nabla^{p, \infty} a_{j, \alpha}\right\|_{\infty} \in \mathscr{E}^{(s)} \quad(j+|\alpha| \leqq m \text { and } j<m) .
$$

Then, our $\mathscr{E}^{(s)}$-version of [5, Proposition 4] is given as follows.

Proposition 2. Let $P$ be the operator in (1.1), let $1<s<\infty$, and let $p \in N$. Assume that $P$ satisfies $\left(\mathrm{A}_{k}\right),\left(\mathrm{B}^{\prime}\right)$ and $\left(\mathrm{D}_{p}^{(s)}\right)$, and that $s$ satisfies $(\mathrm{C}-1)$ or $(\mathrm{C}-2)$. Then, there is an $a_{3}>0$ which satisfies the following condition. If $r>a_{3}$, and if $g(t, x) \in C^{\infty}\left((0, T), H^{\infty}\left(\boldsymbol{R}^{n}\right)\right)$ satisfies $\left(t_{t}\right)^{l} g(t, x) \in C^{0}\left([0, T], H^{\infty}\left(\boldsymbol{R}^{n}\right)\right)$ for any $l \in Z_{+}$and $\left\|\nabla_{r}^{p, \infty} g(t)\right\| \in \mathscr{E}^{(s)}$ uniformly on $[0, T]$, then the equation $(4.1)_{r}$ has a unique solution $v(t, x) \in C^{\infty}\left((0, T), H^{\infty}\left(\boldsymbol{R}^{n}\right)\right)$ such that $\left(t_{t}\right)^{l} v(t, x) \in C^{0}([0, T]$, $\left.H^{\infty}\left(\boldsymbol{R}^{n}\right)\right)$ for any $l \in Z_{+}$and $\left\|\nabla_{r}^{p, \infty} \nabla_{k, r}^{m-1} v(t)\right\| \in \mathscr{E}^{(s)}$ uniformly on $[0, T]$. In addition, if $g(t, x)$ satisfies $\operatorname{supp}(g) \subset C_{p}(0, K)$ for some compact subset $K$ of $\boldsymbol{R}^{n}$, $v(t, x)$ also satisfies $\operatorname{supp}(v) \subset C_{\mu}(0, K)$.

Here, $C_{\mu}(0, K)$ is defined by the case $t_{0}=0$ of

$$
C_{\mu}\left(t_{0}, K\right)=\left\{(t, x) \in[0, T] \times R^{n} ; \min _{y \in K}|x-y| \leqq \frac{\lambda_{\max } T^{k-\mu}}{\mu}\left|t^{\mu}-t_{0}^{\mu}\right|\right\},
$$


where $\mu=\min \left\{\kappa_{1}, \cdots, \kappa_{n}\right\}, k=\max \left\{\kappa_{1}, \cdots, \kappa_{n}\right\}$ and $\lambda_{\max }$ is the least upper bound of $\left|\lambda_{i}(t, x, \xi)\right|(1 \leqq i \leqq m)$ on $\left\{(t, x, \xi) \in[0, T] \times \boldsymbol{R}^{n} \times \boldsymbol{R}^{n} ;|\xi|=1\right\}$.

Proof of Proposition 2. Let $\mathscr{J}$ be as in (2.1). Put $Q_{r}(j, \alpha)((j, \alpha) \in \mathscr{J})$ and $L$ as follows:

$$
\begin{aligned}
Q_{r}(j, \alpha) & =(-1) t^{l(j, \alpha)} a_{j, \alpha}(t, x)\left(t \partial_{t}+r\right)^{j} \partial_{x}^{\alpha}, \\
L & =P+\sum_{(j, \alpha) \in \mathcal{g}} Q_{0}(j, \alpha) .
\end{aligned}
$$

Then, $(4.1)_{r}$ is equivalent to

$$
L\left(t^{r} v\right)=t^{r}\left(g+\sum_{(j, \alpha) \in \mathcal{g}} Q_{r}(j, \alpha) v\right) .
$$

Therefore, to solve (4.1) $)_{r}$ we can use the method of successive approximations: first we solve (by [5, Lemma 11])

$$
\begin{aligned}
& L\left(t^{r} v_{0}\right)=t^{r} g, \\
& L\left(t^{r} v\left(\left(j_{1}, \alpha_{(1)}\right)\right)\right)=t^{r} Q_{r}\left(j_{1}, \alpha_{(1)}\right) v_{0}, \\
& L\left(t^{r} v\left(\left(j_{1}, \alpha_{(1)}\right),\left(j_{2}, \alpha_{(2)}\right)\right)\right)=t^{r} Q_{r}\left(j_{2}, \alpha_{(2)}\right) v\left(\left(j_{1}, \alpha_{(1)}\right)\right), \\
& \quad \vdots \\
& L\left(t^{r} v\left(\left(j_{1}, \alpha_{(1)}\right), \cdots,\left(j_{k}, \alpha_{(k)}\right)\right)\right)=t^{r} Q_{r}\left(j_{k}, \alpha_{(k)}\right) v\left(\left(j_{1}, \alpha_{(1)}\right), \cdots,\left(j_{k-1}, \alpha_{(k-1)}\right)\right),
\end{aligned}
$$

and then we show the convergence of the formal solution

$$
v=v_{0}+\sum_{k=1}^{\infty} \sum_{\substack{\left.j_{i}, \alpha_{(i)}\right) \in \mathscr{G} \\ i=1, \ldots, k}} v\left(\left(j_{1}, \alpha_{(1)}\right), \cdots,\left(j_{k}, \alpha_{(k)}\right)\right) .
$$

The proof of the convergence is as follows. By $\left(\mathrm{D}_{p}^{(s)}\right)$, we have

$$
\left\|\nabla^{p, \infty} L\right\|_{\infty}, \quad\left\|\nabla^{p, \infty} a_{j, \alpha}\right\|_{\infty} \ll B_{0} \theta_{(s)}\left(\rho ; C_{0}(\varepsilon)\right)
$$

for some $B_{0}>0$ and some positive-valued function $C_{0}(\varepsilon)$ in $\varepsilon>0$ satisfying $C_{0}(1)=1$. Since $\left\|\nabla_{r}^{p, \infty} g(t)\right\| \in \mathscr{E}^{(s)}$ uniformly on $[0, T]$, by Lemma 3 we can find a positive-valued function $C(\varepsilon)$ in $\varepsilon>0$ such that

$$
\left\|\nabla_{r}^{p, \infty} g(t)\right\| \ll \theta_{(s)}(\rho ; C(\varepsilon)) \ll \theta_{(s)}(2 \rho ; C(\varepsilon)) \quad \text { on }[0, T]
$$

and that $\theta_{(s)}(\rho ; C(\varepsilon))$ satisfies $\left(\mathrm{M}_{(s)}\right)$ with respect to $C_{0}(\varepsilon)$. Therefore, by the same argument as in [5, formula (7.8) or (7.10)] we have the following: there are $C>0, H>0$ and $a>0$ such that for any $J_{k}=\left(\left(j_{1}, \alpha_{(1)}\right), \cdots,\left(j_{k}, \alpha_{(k)}\right)\right) \in \mathscr{J}^{k}$ we have

$$
\begin{aligned}
& \left\|\left.\right|_{r} ^{p, \infty} \nabla_{k, r}^{m-1} v\left(J_{k}\right)(t)\right\| \ll C^{k+1} t^{l_{1}+\cdots+l_{k}-\left\langle k, \beta_{(1)}+\cdots+\beta_{(k)}\right\rangle} \\
& \quad \times \frac{1}{a_{k+1}} \times \frac{1}{\left(a_{1}\right)^{m-j_{1}-\left|\beta_{(1)}\right|} \times \cdots \times\left(a_{k}\right)^{m-j_{k}-\left|\beta_{(k)}\right|}} \\
& \quad \times \partial_{\rho}^{\left(\left|\alpha_{(1)}\right|-\left|\beta_{(1)}\right|\right)+\cdots+\left(\left|\alpha_{(k)}\right|-\left|\beta_{(k)}\right|\right)} \theta_{(s)}(H \rho ; C(\varepsilon))
\end{aligned}
$$


on $[0, T]$ for any $\beta\left(J_{k}\right)=\left(\beta_{(1)}, \cdots, \beta_{(k)}\right) \in \mathscr{M}\left(J_{k}\right)$, where we put $l_{i}=l\left(j_{i}, \alpha_{(i)}\right)$ $(i=1, \cdots, k), a_{1}=a$ and

$$
a_{i+1}=a+l_{1}+\cdots+l_{i}-\left\langle\kappa, \beta_{(1)}+\cdots+\beta_{(i)}\right\rangle \quad(i=1, \cdots, k) .
$$

Here, we note the following:

$$
\begin{aligned}
& \partial_{\rho}^{\left(\left|\alpha_{(1)}\right|-\left|\beta_{(1)}\right|\right)+\cdots+\left(\left|\alpha_{(k)}\right|-\left|\beta_{(k)}\right|\right)} \theta_{(s)}(H \rho ; C(\varepsilon)) \\
& \ll C(\varepsilon)\left(\varepsilon 2^{s} H\right)^{\left(\left|\alpha_{(1)}\right|-\left|\beta_{(1)}\right|\right)+\cdots+\left(\left|\alpha_{(k)}\right|-\left|\beta_{(k)}\right|\right)} \\
& \times\left[\left(\left|\alpha_{(1)}\right|-\left|\beta_{(1)}\right|\right)+\cdots+\left(\left|\alpha_{(k)}\right|-\mid \beta_{(k)}\right)\right] !{ }^{s} \theta_{s}\left(\varepsilon 2^{s} H \rho\right)
\end{aligned}
$$

holds for any $\varepsilon>0$. Therefore, by (4.5), (4.6) and (2.7) we have

$$
\left\|\nabla_{r}^{p, \infty} \nabla_{n, r}^{m-1} v\left(J_{k}\right)(t)\right\| \ll C(\varepsilon) F_{a}\left(J_{k}, \beta\left(J_{k}\right), C, \varepsilon R, T\right) \theta_{s}(\varepsilon R \rho)
$$

(where $R=2^{s} H$ ) for any $\varepsilon>0$ and $\beta\left(J_{k}\right) \in \mathscr{M}\left(J_{k}\right)$, that is,

$$
\sum_{k=1}^{\infty} \sum_{J_{k} \in \xi^{k}}\left\|\nabla_{r}^{p, \infty} \nabla_{x, r}^{m-1} v\left(J_{k}\right)(t)\right\| \ll C(\varepsilon) M_{a}\left(\left\{\beta\left(J_{k}\right)\right\}, C, \varepsilon R, T\right) \theta_{s}(\varepsilon R \rho)
$$

for any $\varepsilon>0$ (by (2.8)). Hence, by the condition (I-2) in Proposition 1 we obtain

$$
\sum_{k=1}^{\infty} \sum_{J_{k} \in \mathcal{g}^{k}}\left\|\nabla_{r}^{p, \infty} \nabla_{\kappa, r}^{m-1} v\left(J_{k}\right)(t)\right\| \in \mathscr{E}^{\mathscr{( s )}} \quad \text { uniformly on }[0, T]
$$

Thus, the existence part of Proposition 2 is proved. The other part may be proved in the same way.

Q.E.D.

\section{§ 5. Proof of (II): Asymptotic expansions}

To have the part (II) in Theorems 1 and 2 , it is sufficient to establish the $\mathscr{E}^{(s)}$-version of [5, Proposition 5] (see also [5, §§ 3 and 8]).

Let $P$ be the operator in (1.1), and let us consider

$$
\left\{\begin{array}{l}
P u=f, \\
\left.\partial_{t}^{i} u\right|_{t=T}=0 \quad \text { for } i=0,1, \cdots, m-1,
\end{array}\right.
$$

where $u=u(t, x)$ and $f=f(t, x)$. Then, our $\mathscr{E}^{(s)}$-version of [5, Proposition 5] is given as follows.

Proposition 3. Let $P$ be the operator in (1.1), let $1<s<\infty$, and let $p \in N$. Assume that $P$ satisfies $\left(\mathrm{A}_{\kappa}\right),\left(\mathrm{B}^{\prime}\right)$ and $\left(\mathrm{D}_{p}^{(s)}\right)$, and that $s$ satisfies $(\mathrm{C}-1)$ or $(\mathrm{C}-2)$. Then, if $f(t, x) \in C^{\infty}\left((0, T], H^{\infty}\left(\boldsymbol{R}^{n}\right)\right)$ satisfies $\left.\partial_{t}^{i} f\right|_{t=T}=0$ for $i=0,1, \cdots, p-1$ and $t^{A}\left\|\nabla_{0}^{p, \infty} f(t)\right\| \in \mathscr{E}^{\mathscr{C}(s)}$ uniformly on $(0, T]$ for some $A>0$, then the equation (5.1) has a unique solution $u(t, x) \in C^{\infty}\left((0, T], H^{\infty}\left(\boldsymbol{R}^{n}\right)\right)$ such that $\left.\partial_{t}^{i} u\right|_{t=T}=0$ for 
$i=0,1, \cdots, m+p-1$ and that $t^{B}\left\|\nabla_{0}^{p, \infty} \nabla_{k, 0}^{m-1} u(t)\right\| \in \mathscr{E}^{(s)}$ uniformly on $(0, T]$ for some $B>0$. In addition, if $f(t, x)$ satisfies $\operatorname{supp}(f) \subset C_{\mu}(T, K)$ for some compact subset $K$ of $\boldsymbol{R}^{n}, u(t, x)$ also satisfies $\operatorname{supp}(u) \subset C_{\mu}(T, K)$. (Here, $C_{\mu}(T, K)$ is defined by the case $t_{0}=T$ of (4.2).)

Proof. Let $Q_{r}(j, \alpha)$ and $L$ be as in (4.3). Then, to solve (5.1) we can use the method of successive approximations as follows: first we solve

$$
\begin{aligned}
& \left\{\begin{array}{l}
L u_{0}=f, \\
\left.\partial_{t}^{i} u_{0}\right|_{t=T}=0 \quad \text { for } i=0,1, \cdots, m+p-1,
\end{array}\right. \\
& \left\{\begin{array}{l}
L u\left(\left(j_{1}, \alpha_{(1)}\right)\right)=Q_{0}\left(j_{1}, \alpha_{(1)}\right) u_{0}, \\
\left.\partial_{t}^{i} u\left(\left(j_{1}, \alpha_{(1)}\right)\right)\right|_{t=T}=0 \quad \text { for } i=0,1, \cdots, m+p-1, \\
\vdots
\end{array}\right. \\
& \begin{cases}L u\left(\left(j_{1}, \alpha_{(1)}\right), \cdots,\left(j_{k}, \alpha_{(k)}\right)\right)=Q_{0}\left(j_{k}, \alpha_{(k)}\right) u\left(\left(j_{1}, \alpha_{(1)}\right), \cdots,\left(j_{k-1}, \alpha_{(k-1)}\right)\right), \\
\left.\partial_{t}^{i} u\left(\left(j_{1}, \alpha_{(1)}\right), \cdots,\left(j_{k}, \alpha_{(k)}\right)\right)\right|_{t=T}=0 & \text { for } i=0,1, \cdots, m+p-1,\end{cases}
\end{aligned}
$$

and then we show the convergence of the formal solution

$$
u=u_{0}+\sum_{k=1}^{\infty} \sum_{\substack{\left.j_{i}, \alpha_{(i)}\right) \in \mathcal{F} \\ i=1, \ldots, k}} u\left(\left(j_{1}, \alpha_{(1)}\right), \cdots,\left(j_{k}, \alpha_{(k)}\right)\right) .
$$

The proof of the convergence is as follows. Since $\left(\mathrm{D}_{p}^{(s)}\right)$ and $t^{A}\left\|\nabla_{0}^{p, \infty} f(t)\right\|$ $\in \mathscr{E}^{(s)}$ (uniformly on $(0, T]$ ) are assumed, we have

$$
\begin{aligned}
& \left\|\nabla^{p, \infty} L\right\|_{\infty},\left\|\nabla^{p, \infty} a_{j, \alpha}\right\|_{\infty} \ll B_{0} \theta_{(s)}\left(\rho ; C_{0}(\varepsilon)\right), \\
& \left\|V_{0}^{p, \infty} f(t)\right\| \ll t^{-A} \theta_{(s)}(2 \rho ; C(\varepsilon)) \quad \text { on }(0, T]
\end{aligned}
$$

for some $B_{0}>0, C_{0}(\varepsilon)$ and $C(\varepsilon)$ such that $\theta_{(s)}(\rho ; C(\varepsilon))$ satisfies $\left(\mathrm{M}_{(s)}\right)$ with respect to $C_{0}(\varepsilon)$. Therefore, by the same argument as in (4.5) (see also [5, $\S \S 5$ and 8]) we can obtain the following: there are $C>0, H>0$ and $a>0$ such that for any $J_{k}=\left(\left(j_{1}, \alpha_{(1)}\right), \cdots,\left(j_{k}, \alpha_{(k)}\right)\right) \in \mathscr{J}^{k}$ we have

$$
\begin{aligned}
\| \nabla_{0}^{p, \infty} & V_{k, 0}^{m-1} u\left(J_{k}\right)(t) \| \ll C^{k+1} t^{-A}\left(\frac{T}{t}\right)^{a+1} T^{l_{1}+\cdots+l_{k}-\left\langle k, \beta_{(1)}+\cdots+\beta_{(k)}\right\rangle} \\
& \times \frac{1}{a+b_{1}} \times \frac{1}{\left(b_{1}\right)^{m-j_{1}-\left|\beta_{(1)}\right|} \times \cdots \times\left(b_{k}\right)^{m-j_{k}-\left|\beta_{(k)}\right|}} \\
& \times \partial_{\rho}^{\left(\left|\alpha_{(1)}\right|-\left|\beta_{(1)}\right|\right)+\cdots+\left(\left|\alpha_{(k)}\right|-\mid \beta_{(k)}\right) \mid} \theta_{(s)}(H \rho ; C(\varepsilon))
\end{aligned}
$$

on $(0, T]$ for any $\left(\beta_{(1)}, \cdots, \beta_{(k)}\right) \in Z_{+}^{n} \times \cdots \times Z_{+}^{n}$ satisfying

$$
\left\{\begin{array}{l}
(0, \cdots, 0) \leqq \beta_{(i)} \leqq \alpha_{(i)} \quad(i=1, \cdots, k), \\
\left\langle k, \beta_{(i)}+\cdots+\beta_{(k)}\right\rangle \leqq l_{i}+\cdots+l_{k} \quad(i=1, \cdots, k),
\end{array}\right.
$$

where we put $l_{i}=l\left(j_{i}, \alpha_{(i)}\right)(i=1, \cdots, k)$ and 


$$
b_{i}=1+l_{i}+\cdots+l_{k}-\left\langle\kappa, \beta_{(i)}+\cdots+\beta_{(k)}\right\rangle \quad(i=1, \cdots, k) .
$$

Hence, by an argument quite parallel to the proof of Proposition 2 we obtain

$$
t^{a+1+A} \sum_{k=1}^{\infty} \sum_{J_{k} \in \mathcal{F}^{k}}\left\|\nabla_{0}^{p, \infty} \nabla_{k, 0}^{m-1} u\left(J_{k}\right)(t)\right\| \in \mathscr{E}^{\mathscr{E}(s)} \quad \text { uniformly on }(0, T] .
$$

Thus, the existence part of Proposition 3 is proved. The other part may be proved in the same way.

Q.E.D.

\section{§. Proof of Proposition 1}

Note that "(I-2) $\Rightarrow(\mathrm{I}-3)$ " is trivial, that "(II-1) $\Rightarrow(\mathrm{II}-2)$ " was already proved in [5], and that "(II-2) $\Rightarrow$ (II-3)" is trivial. Therefore, to have Proposi. tion 1 it is sufficient to prove the following parts: "(I-1) $\Rightarrow(\mathrm{I}-2)$ ", "(I-3) $\Rightarrow(\mathrm{I}-1)$ " and "(II-3) $\Rightarrow($ II- 1$)$ ".

For $z \geqq 0$, we write

$$
z !=\Gamma(1+z)
$$

where $\Gamma(z)$ is the gamma-function of Euler. Then, we have

Lemma 5. (1) For any $p, q>0$ we have

$$
\frac{1}{1+p+q} \leqq \frac{(p+q) !}{p ! q !} \leqq 2^{p+q}
$$

(2) For any $p_{1}, p_{2}, \cdots, p_{k}>0$ we have

$$
1 \leqq \frac{\left(1+p_{1}+p_{2}+\cdots+p_{k}\right)^{1+p_{1}+p_{2}+\cdots+p_{k}}}{\left(1+p_{1}\right)^{p_{1}}\left(1+p_{1}+p_{2}\right)^{p_{2}} \cdots\left(1+p_{1}+p_{2}+\cdots+p_{k}\right)^{p_{k}}} \leqq e^{p_{1}+p_{2}+\cdots+p_{k}} .
$$

(3) For any $0<a<b$, there are $A_{1}>0, A_{2}>0, C_{1}>0$ and $C_{2}>0$ which satisfy the following: for any $p_{1}, p_{2}, \cdots, p_{k}>0$ satisfying $a \leqq p_{i} \leqq b(i=1,2, \cdots, k)$ we have

$$
A_{1} C_{1}^{k} \leqq \frac{\left(p_{1}+p_{2}+\cdots+p_{k}\right) !}{(1)^{p_{1}}(2)^{p_{2}} \cdots(k)^{p_{k}}} \leqq A_{2} C_{2}^{k}
$$

In $[5, \S 4]$, we gave an interpretation of our irregularity condition. The following lemma is easily obtained by the proof of [5, Proposition 1]. Let $\mathscr{J}$, $l(j, \alpha)$ and $\kappa=\left(\kappa_{1}, \cdots, \kappa_{n}\right)$ be as in $\S 2$.

Lemma 6. Let $(j, \alpha) \in \mathscr{J}$, let $1 \leqq s<\infty$ and let $\sigma_{j, \alpha}(\geqq 1)$ be as in (2.2). Then, we have the following results.

(1) $\sigma_{j, \alpha}=1$ is equivalent to the condition $l(j, \alpha) \geqq\langle\kappa, \alpha\rangle$.

(2) When $1 \leqq s<\sigma_{j, \alpha} /\left(\sigma_{j, \alpha}-1\right)$, there is a $z \in \boldsymbol{R}^{n}$ such that $(0, \cdots, 0) \leqq$ 
$z \leqq \alpha, 0<|z|<|\alpha|,\langle\kappa, z\rangle \leqq l(j, \alpha)$ and

$$
s<\frac{m-j-|z|}{|\alpha|-|z|} .
$$

(3) When $s=\sigma_{j, \alpha} /\left(\sigma_{j, \alpha}-1\right)$, there is a $z \in \boldsymbol{R}^{n}$ such that $(0, \cdots, 0) \leqq z \leqq \alpha$, $0<|z|<|\alpha|,\langle\kappa, z\rangle=l(j, \alpha)$ and

$$
s=\frac{m-j-|z|}{|\alpha|-|z|}
$$

Moreover, we can find $\tau \in \mathbb{S}_{n}$ and $p \in\{1, \cdots, n\}$ such that $\kappa_{\tau(1)} \leqq \kappa_{\tau(2)} \leqq \cdots \leqq$ $\kappa_{\tau(n)}, z_{\tau(k)}=\alpha_{z(k)}$ for $1 \leqq k<p, 0<z_{\tau(p)} \leqq \alpha_{\tau(p)}$, and $z_{\tau(k)}=0$ for $p<k \leqq n$ (where $z=$ $\left(z_{1}, \cdots, z_{n}\right)$ and $\left.\alpha=\left(\alpha_{1}, \cdots, \alpha_{n}\right)\right)$.

Here, we used the following notations for $a=\left(a_{1}, \cdots, a_{n}\right)$ and $b=\left(b_{1}, \cdots\right.$, $\left.b_{n}\right): a \leqq b$ means that $a_{i} \leqq b_{i}$ holds for any $i,|a|=\left|a_{1}\right|+\cdots+\left|a_{n}\right|$ and $\langle a, b\rangle=$ $a_{1} b_{1}+\cdots+a_{n} b_{n}$.

To prove Proposition 1, we need further information on $z$. Let us give a refinement of Lemma 6 . For $(j, \alpha) \in \mathscr{J}$, denote by $\mathscr{Z}(j, \alpha)$ the set of all $z \in \boldsymbol{R}^{n}$ satisfying the following:

$$
\left\{\begin{array}{l}
(0, \cdots, 0) \leqq z \leqq \alpha \\
|z|<|\alpha| \\
\langle\kappa, z\rangle \leqq l(j, \alpha)
\end{array}\right.
$$

Let $\kappa=\left(\kappa_{1}, \cdots, \kappa_{n}\right)$ be the one in $\left(\mathrm{A}_{\kappa}\right)$. Define $p \sim q$ by $\kappa_{p}=\kappa_{q}$. Then, the $\sim$ defines an equivalence relation in $\{1,2, \cdots, n\}$; therefore, we obtain $K_{1}$. $\cdots, K_{\nu} \subset\{1,2, \cdots, n\}$ satisfying the following: $K_{1} \cup \cdots \cup K_{\nu}=\{1,2, \cdots, n\}$, $K_{i} \neq \varnothing, K_{i} \cap K_{j}=\varnothing$ for $i \neq j$, and

$$
\begin{cases}\kappa_{p}=\kappa_{q} & \text { for } p, q \in K_{i}, \\ \kappa_{p} \neq \kappa_{q} & \text { for } p \in K_{i}, q \in K_{j} \text { and } i \neq j .\end{cases}
$$

Under this notation, for $x=\left(x_{1}, \cdots, x_{n}\right)$ we write

$$
|x|_{i}=\sum_{p \in K_{i}}\left|x_{p}\right| \quad(i=1, \cdots, \nu) .
$$

Then, our refinement of Lemma 6 is given as follows. Let $S_{\kappa}(\alpha)$ be as in (2.5).

Lemma 7. Let $(j, \alpha) \in \mathscr{J}$, let $1 \leqq s<\infty$ and let $\kappa=\left(\kappa_{1}, \cdots, \kappa_{n}\right)$ be the one in $\left(\mathrm{A}_{\kappa}\right)$. Then, we have the following results.

(1) When $1 \leqq s<\sigma_{j, \alpha} /\left(\sigma_{j, \alpha}-1\right)$, there is a $z \in \mathscr{Z}(j, \alpha)$ such that 


$$
s<\frac{m-j-|z|}{|\alpha|-|z|}
$$

and that $0<|z|_{i}<|\alpha|_{i}$ holds for some $i \in\{1, \cdots, \nu\}$. that

(2) When $s=\sigma_{j, \alpha} /\left(\sigma_{j, \alpha}-1\right)$ and $l(j, \alpha) \oplus S_{k}(\alpha)$, there is a $z \in \mathscr{Z}(j, \alpha)$ such

$$
s=\frac{m-j-|z|}{|\alpha|-|z|}
$$

and that $0<|z|_{i}<|\alpha|_{i}$ holds for some $i \in\{1, \cdots, \nu\}$. that

(3) When $s=\sigma_{j, \alpha} /\left(\sigma_{j, \alpha}-1\right)$ and $l(j, \alpha) \in S_{\kappa}(\alpha)$, there is a $z \in \mathscr{Z}(j, \alpha)$ such

$$
s=\frac{m-j-|z|}{|\alpha|-|z|}
$$

and that $|z|_{i}=0$ or $|z|_{i}=|\alpha|_{i}$ holds for any $i \in\{1, \cdots, \nu\}$.

(4) When $s>\sigma_{j, \alpha} /\left(\sigma_{j, \alpha}-1\right)$, there are no $z \in \mathscr{Z}(j, \alpha)$ such that

$$
s \leqq \frac{m-j-|z|}{|\alpha|-|z|}
$$

Proof. Note that $\kappa_{i}>0(1 \leqq i \leqq n)$ and $l(j, \alpha)>0$ are assumed (as in $\left.\S 2\right)$. Put $\alpha=\left(\alpha_{1}, \cdots, \alpha_{n}\right)$.

When $1 \leqq s<\sigma_{j, \alpha} /\left(\sigma_{j, \alpha}-1\right)$, by (2) in Lemma 6 we can find a $w=$ $\left(w_{1}, \cdots, w_{n}\right) \in \boldsymbol{R}^{n}$ such that $(0, \cdots, 0) \leqq w \leqq \alpha, 0<|w|<|\alpha|,\langle\kappa, w\rangle \leqq l(j, \alpha)$ and

$$
s<\frac{m-j-|w|}{|\alpha|-|w|} .
$$

Then, we can obtain (1) in Lemma 7 by defining $z=\left(z_{1}, \cdots, z_{n}\right) \in \boldsymbol{R}^{n}$ as follows: when $w_{k}=0$, we put $z_{k}=0$; when $w_{k}>0$, we choose $z_{k} \in \boldsymbol{R}$ so that $0<\boldsymbol{z}_{k}<w_{k}$ and that $z_{k}$ is sufficiently close to $w_{k}$. In fact, if $z \in \boldsymbol{R}^{n}$ is as above, $z \in \mathscr{Z}(j, \alpha)$ is clear, $(6.2)$ is verified by the fact that

$$
\frac{m-j-|z|}{|\alpha|-|z|} \rightarrow \frac{m-j-|w|}{|\alpha|-|w|}
$$

as $|z| \rightarrow|w|$, and the last condition is verified as follows. Since $|w|>0$, we have $w_{k}>0$ for some $k$ and therefore $0<z_{k}<w_{k} \leqq \alpha_{k}$; hence by taking $i \epsilon$ $\{1, \cdots, \nu\}$ such that $k \in K_{i}$ we obtain $0<|z|_{i}<|\alpha|_{i}$. Thus, (1) is proved.

When $s=\sigma_{j, \alpha} /\left(\sigma_{j, \alpha}-1\right)$, by (3) in Lemma 6 we can find $z=\left(z_{1}, \cdots, z_{n}\right)$ $\in \boldsymbol{R}^{n}, \tau \in \mathfrak{S}_{n}$ and $p \in\{1, \cdots, n\}$ such that $(0, \cdots, 0) \leqq z \leqq \alpha, 0<|z|<|\alpha|,\langle\kappa, z\rangle$ $=l(j, \alpha)$, 


$$
s=\frac{m-j-|z|}{|\alpha|-|z|}
$$

$\kappa_{\imath(1)} \leqq \kappa_{\tau(2)} \leqq \cdots \leqq \kappa_{\tau(n)}, \quad z_{\tau(k)}=\alpha_{\tau(k)}$ for $1 \leqq k<p, 0<z_{\tau(p)} \leqq \alpha_{\imath(p)}$, and $z_{\tau(k)}=0$ for $p<k \leqq n$. Take $i_{0} \in\{1, \cdots, \nu\}$ such that $\tau(p) \in K_{i_{0}}$. Then we have

$$
\left\{\begin{array}{l}
0<|z|_{i_{0}} \leqq|\alpha|_{i_{0}} \\
|z|_{h}=0 \text { or } \quad|z|_{h}=|\alpha|_{h} \quad \text { for } h \neq i_{0} .
\end{array}\right.
$$

Therefore, to have (2) and (3) in Lemma 7 it is sufficient to prove the following fact:

$$
\begin{aligned}
& |z|_{i_{0}}<|\alpha|_{i_{0}}, \quad \text { when } l(j, \alpha) \oplus S_{\kappa}(\alpha), \\
& |z|_{i_{0}}=|\alpha|_{i_{0}}, \quad \text { when } l(j, \alpha) \in S_{\kappa}(\alpha) .
\end{aligned}
$$

Note that we have the condition $l(j, \alpha)<\langle\kappa, \alpha\rangle$, since $s=\sigma_{j, \alpha} /\left(\sigma_{j, \alpha}-1\right)<\infty$ and therefore $\sigma_{j, \alpha}>1$ (see (1) in Lemma 6).

Let us show (6.3). Assume that $l(j, \alpha) \oplus S_{x}(\alpha)$. When $0<z_{\tau(p)}<\alpha_{\tau(p)}$, (6.3) is trivial. Therefore, in the discussion below we may assume that $z_{\tau(p)}=\alpha_{\tau(p)}$. In this case, by the condition $l(j, \alpha)=\langle\kappa, z\rangle$ we have

$$
l(j, \alpha)=\kappa_{\tau(1)} \alpha_{\tau(1)}+\cdots+\kappa_{\tau(p)} \alpha_{\tau(p)} .
$$

Moreover, we can see the following: $p \leqq n-1, \kappa_{\tau(p)}=\kappa_{\tau(p+1)}$ and $\alpha_{\tau(k)}>0$ for some $k \in\{p+1, \cdots, n\}$. In fact, these are verified as follows: if $p=n$, (6.5) contradicts the condition $l(j, \alpha)<\langle\kappa, \alpha\rangle$; if $\kappa_{\tau(p)}<\kappa_{\tau(p+1)}$, (6.5) contradicts the condition $l(j, \alpha) \notin S_{\kappa}(\alpha)$; if $\alpha_{\tau(p+1)}=\cdots=\alpha_{\tau(n)}=0,(6.5)$ contradicts the condition $l(j, \alpha)<\langle\kappa, \alpha\rangle$. Hence, by putting

$$
q=\min \left\{k \in\{p+1, \cdots, n\} ; \alpha_{\tau(k)}>0\right\}
$$

we have $\alpha_{\tau(q)}>0, \alpha_{\tau(k)}=0$ for $p+1 \leqq k<q$ and

$$
l(j, \alpha)=\kappa_{\tau(1)} \alpha_{\tau(1)}+\cdots+\kappa_{\tau(h)} \alpha_{\tau(h)}
$$

for any $h \in\{p, \cdots, q-1\}$. If $\kappa_{z(h)}<\kappa_{z(h+1)}$ holds for some $h \in\{p, \cdots, q-1\},(6.6)$ contradicts the condition $l(j, \alpha) \notin S_{\kappa}(\alpha)$; therefore we have $\kappa_{\tau(p)}=\kappa_{\tau(p+1)}=\cdots$ $=\kappa_{\tau(q)}$. This implies that $\tau(k) \in K_{i_{0}}$ for $k=p, p+1, \cdots, q$. Since $z_{\tau(q)}=0$ and $\alpha_{\imath(q)}>0$, we obtain

$$
|\alpha|_{i_{0}}-|z|_{i_{0}} \geqq \alpha_{\tau(q)}-z_{\tau(q)}=\alpha_{\tau(q)}>0
$$

which proves (6.3). As a consequence, we obtain (2) in Lemma 7.

Let us next show (6.4). Assume that $l(j, \alpha) \in S_{k}(\alpha)$. Then, there are $\nu \in \mathbb{S}_{n}$ and $q \in\{1, \cdots, n-1\}$ such that 


$$
\begin{aligned}
& l(j, \alpha)=\kappa_{\nu(1)} \alpha_{\nu(1)}+\cdots+\kappa_{\nu(q)} \alpha_{\nu(q)}, \\
& \left\{\kappa_{\nu(1)}, \cdots, \kappa_{\nu(q)}\right\}<\left\{\kappa_{\nu(q+1)}, \cdots, \kappa_{\nu(n)}\right\} .
\end{aligned}
$$

Since $\kappa_{\imath(1)} \leqq \kappa_{\tau(2)} \leqq \cdots \leqq \kappa_{\tau(n)}$ is assumed, by (6.8) we have $\{\nu(1), \cdots, \nu(q)\}=$ $\{\tau(1), \cdots, \tau(q)\}$ and therefore

$$
\begin{aligned}
& l(j, \alpha)=\kappa_{\tau(1)} \alpha_{\tau(1)}+\cdots+\kappa_{\tau(q)} \alpha_{\tau(q)}, \\
& \left\{\kappa_{\tau(1)}, \cdots, \kappa_{\tau(q)}\right\}<\left\{\kappa_{\tau(q+1)}, \cdots, \kappa_{\tau(n)}\right\} .
\end{aligned}
$$

On the other hand, by the condition $l(j, \alpha)=\langle\kappa, z\rangle$ and by the choice of $z$ we have

$$
l(j, \alpha)=\kappa_{\tau(1)} \alpha_{\tau(1)}+\cdots+\kappa_{\tau(p-1)} \alpha_{\tau(p-1)}+\kappa_{\tau(p)} z_{\tau(p)} .
$$

If $q<p$, by (6.9) and (6.11) we have

$$
\kappa_{\tau(q+1)} \alpha_{\tau(q+1)}+\cdots+\kappa_{\tau(p-1)} \alpha_{z(p-1)}+\kappa_{\tau(p)} z_{\tau(p)}=0
$$

and therefore we have $z_{z(p)}=0$; this contradicts the condition $0<z_{\tau(p)} \leqq \alpha_{\tau(p)}$. Hence, we may assume that $q \geqq p$. In this case, by (6.9) and (6.11) we have

$$
\kappa_{\tau(p)}\left(\alpha_{\tau(p)}-z_{\tau(p)}\right)+\kappa_{\tau(p+1)} \alpha_{\tau(p+1)}+\cdots+\kappa_{\tau(q)} \alpha_{\tau(q)}=0 ;
$$

therefore, we have $\alpha_{\tau(p)}=z_{\tau(p)}$ and $\alpha_{\tau(k)}=0 \quad\left(=z_{\tau(k)}\right)$ for $p<k \leqq q$. Thus, by combining this with the known condition $\kappa_{\tau(q)}<\kappa_{\tau(q+1)}$ (by $\left.(6.10)\right)$ we obtain

$$
z_{\tau(k)}=\alpha_{\tau(k)} \quad \text { for any } \tau(k) \in K_{i_{0}}
$$

which proves (6.4). As a consequence, we obtain (3) in Lemma 7.

Lastly, let us prove (4) in Lemma 7 by showing the following: under the conditions $s>\sigma_{j, \alpha} /\left(\sigma_{j, \alpha}-1\right), z \in \mathscr{Z}(j, \alpha)$ and

$$
s \leqq \frac{m-j-|z|}{|\alpha|-|z|},
$$

we can obtain a contradiction. Assume that $s>\sigma_{j, \alpha} /\left(\sigma_{j, \alpha}-1\right)$ and that $z=$ $\left(z_{1}, \cdots, z_{n}\right) \in \mathscr{Z}(j, \alpha)$ satisfies (6.12). Then, we have

$$
\sigma_{j, \alpha} /\left(\sigma_{j, \alpha}-1\right)<\frac{m-j-|z|}{|\alpha|-|z|} .
$$

Since $\sigma_{j, \alpha}>1$, by $(6.13)$ we have

$$
\frac{m-j-|z|}{m-j-|\alpha|}<\min _{\tau \in \Phi_{n}}\left(\max _{1 \leqq r \leqq n} M_{j, \alpha}(\tau, r)\right) .
$$

Therefore, for any $\tau \in \mathbb{S}_{n}$ we can find a $p \in\{1, \cdots, n\}$ such that 


$$
\frac{m-j-|z|}{m-j-|\alpha|}<M_{j, \alpha}(\tau, p)
$$

and this is equivalent to

$$
l(j, \alpha)<\sum_{i=1}^{p}\left(\kappa_{\tau(i)}-\kappa_{\tau(p)}\right) \alpha_{\tau(i)}+\kappa_{\tau(p)} \sum_{i=1}^{n} z_{\tau(i)} .
$$

On the other hand, by the condition $\langle\kappa, z\rangle \leqq l(j, \alpha)$ we have

$$
\sum_{i=1}^{n} \kappa_{\tau(i)} z_{\tau(i)} \leqq l(j, \alpha)
$$

Hence, by (6.14) and (6.15) we obtain

$$
\sum_{i=1}^{p}\left(\kappa_{\tau(p)}-\kappa_{\tau(i)}\right)\left(\alpha_{\tau(i)}-z_{\tau(i)}\right)+\sum_{i=p+1}^{n}\left(\kappa_{\tau(i)}-\kappa_{\tau(p)}\right) z_{\tau(i)}<0 .
$$

Thus, by choosing $\tau \in \mathbb{S}_{n}$ so that $\kappa_{\tau(1)} \leqq \kappa_{\tau(2)} \leqq \cdots \leqq \kappa_{\tau(n)}$ we can obtain a contradiction from (6.16).

Q.E.D.

Corollary to (3) IN Lemma 7. Assume that $s=\sigma_{j, \alpha} /\left(\sigma_{j, \alpha}-1\right)$ and $l(j, \alpha) \in S_{\kappa}(\alpha)$ hold. Let $\nu \in \mathbb{S}_{n}$ and $q \in\{1, \cdots, n-1\}$ be such that

$$
\left\{\begin{array}{l}
l(j, \alpha)=\kappa_{\nu(1)} \alpha_{\nu(1)}+\cdots+\kappa_{\nu(q)} \alpha_{\nu(q)}, \\
\left\{\kappa_{\nu(1)}, \cdots, \kappa_{\nu(q)}\right\}<\left\{\kappa_{\nu(q+1)}, \cdots, \kappa_{\nu(n)}\right\} .
\end{array}\right.
$$

Then, $z^{*}=\left(z_{1}^{*}, \cdots, z_{n}^{*}\right) \in Z_{+}^{n}$ defined by

$$
z_{\nu(k)}^{*}= \begin{cases}\alpha_{\nu(k)}, & \text { for } 1 \leqq k \leqq q, \\ 0, & \text { for } q+1 \leqq k \leqq n\end{cases}
$$

satisfies $z^{*} \in \mathscr{Z}(j, \alpha),\left\langle\kappa, z^{*}\right\rangle=l(j, \alpha)$ and

$$
s=\frac{m-j-\left|z^{*}\right|}{|\alpha|-\left|z^{*}\right|} .
$$

Proof. In the proof of (3) in Lemma 7 we already have the following: $\{\nu(1), \cdots, \nu(q)\}=\{\tau(1), \cdots, \tau(q)\},\{\nu(q+1), \cdots, \nu(n)\}=\{\tau(q+1), \cdots, \tau(n)\}, z_{\tau(k)}$ $=\alpha_{\tau(k)}$ for $1 \leqq k \leqq q$, and $z_{\tau(k)}=0$ for $q+1 \leqq k \leqq n$. Hence, we obtain $z=z^{*}$.

Q.E.D.

Note that the condition "(C-1) or (C-2)" is equivalent to the following: for any $(j, \alpha) \in \mathscr{J}$ we have the condition (i) or (ii) given below:

(i ) $1<s<\sigma_{j, \alpha} /\left(\sigma_{j, \alpha}-1\right)$,

(ii) $s=\sigma_{j, \alpha} /\left(\sigma_{\hat{\jmath}, \alpha}-1\right)$ and $l(j, \alpha) \notin S_{k}(\alpha)$. 
Hence, by (1) and (2) in Lemma 7 we can obtain the following result which yields a proof of “(I-1) $\Rightarrow(\mathrm{I}-2)$ " in Proposition 1.

Lemma 8. Let $1<s<\infty$, let $l(j, \alpha)>0((j, \alpha) \in \mathscr{J})$ and let $\kappa_{i}>0(1 \leqq i \leqq n)$. Assume that (C-1) or (C-2) holds. Then, there are $c_{1}>0, c_{2}>0$ and $c_{3}>0$ such that for any $J_{k}=\left(\left(j_{1}, \alpha_{(1)}\right), \cdots,\left(j_{k}, \alpha_{(k)}\right)\right) \in \mathscr{J}^{k}$ we can find a $\beta\left(J_{k}\right)=\left(\beta_{(1)}, \cdots\right.$, $\left.\beta_{(k)}\right) \in \mathscr{M}\left(J_{k}\right)$ which satisfies the following conditions (i) $\sim(\mathrm{iii})$ :

(i ) $\left(\left|\alpha_{(1)}\right|-\left|\beta_{(1)}\right|\right)+\cdots+\left(\left|\alpha_{(k)}\right|-\left|\beta_{(k)}\right|\right) \geqq c_{1} k$.

(ii) $\frac{c_{2}+\left(m-j_{1}-\left|\beta_{(1)}\right|\right)+\cdots+\left(m-j_{k}-\left|\beta_{(k)}\right|\right)}{\left(\left|\alpha_{(1)}\right|-\left|\beta_{(1)}\right|\right)+\cdots+\left(\left|\alpha_{(k)}\right|-\left|\beta_{(k)}\right|\right)} \geqq s$.

(iii) There are $I_{1}, \cdots, I_{p} \subset\{1, \cdots, k\}$ and $N_{1}, \cdots, N_{k} \in Z_{+}$such that $p \leqq 2 \nu$, $I_{r} \neq \varnothing, I_{r} \cap I_{q}=\varnothing$ for $r \neq q, I_{1} \cup \cdots \cup I_{p}=\{1, \cdots, k\}, \quad\left\{N_{i} ; i \in I_{r}\right\}=\{0,1, \cdots$, $\left.\left|I_{r}\right|-1\right\}$ for $r=1, \cdots, p$, and

$$
l\left(j_{1}, \alpha_{(1)}\right)+\cdots+l\left(j_{i}, \alpha_{(i)}\right)-\left\langle\kappa, \beta_{(1)}+\cdots+\beta_{(i)}\right\rangle \geqq c_{3} N_{i}
$$

for $i=1, \cdots, k$, where $\nu$ is the one in (6.1) and $\left|I_{r}\right|$ means the number of elements in $I_{r}$.

Proof. Since (C-1) or (C-2) holds, by (1) and (2) in Lemma 7 we have the following: for any $(j, \alpha) \in \mathscr{J}$ we can find a $z(j, \alpha) \in \mathscr{Z}(j, \alpha)$ such that

$$
s \leqq \frac{m-j-|z(j, \alpha)|}{|\alpha|-|z(j, \alpha)|}
$$

and that $0<|z(j, \alpha)|_{i}<|\alpha|_{i}$ holds for some $i \in\{1, \cdots, \nu\}$. We take $z(j, \alpha) \epsilon$ $\mathscr{Z}(j, \alpha)$ (for any $(j, \alpha) \in \mathscr{J})$ as above and fix them hereafter.

Take any $J_{k}=\left(\left(j_{1}, \alpha_{(1)}\right), \cdots,\left(j_{k}, \alpha_{(k)}\right)\right) \in \mathscr{J}^{k}$ and fix it. Put $\alpha_{(i)}=\left(\alpha_{(i), 1}, \cdots\right.$, $\left.\alpha_{(i), n}\right), l\left(j_{i}, \alpha_{(i)}\right)=l_{i}$ and $z\left(j_{i}, \alpha_{(i)}\right)=z_{(i)}=\left(z_{(i), 1}, \cdots, z_{(i), n}\right)(i=1, \cdots, k)$. Then, we have $(0, \cdots, 0) \leqq z_{(i)} \leqq \alpha_{(i)},\left|z_{(i)}\right|<\left|\alpha_{(i)}\right|,\left\langle\kappa, z_{(i)}\right\rangle \leqq l_{i}$,

$$
s \leqq \frac{m-j_{i}-\left|z_{(i)}\right|}{\left|\alpha_{(i)}\right|-\left|z_{(i)}\right|}
$$

and the following condition

$$
0<\left|z_{(i)}\right|_{p_{i}}<\left|\alpha_{(i)}\right|_{p_{i}} \quad \text { for some } p_{i} \in\{1, \cdots, \nu\}
$$

$(i=1, \cdots, k)$. Put $\alpha_{(0)}=(1, \cdots, 1)$ and $\alpha_{(k+1)}=(0, \cdots, 0)$. Let $K_{1}, \cdots, K_{\nu}$ be the ones used in (6.1). Choose $r_{1}, \cdots, r_{\nu} \in\{0,1, \cdots, k\}$ such that

$$
\begin{aligned}
\left|\alpha_{\left(r_{j}+1\right)}\right|_{j}+\cdots+\left|\alpha_{(k+1)}\right|_{j} & \leqq\left|z_{(1)}\right|_{j}+\cdots+\left|z_{(k)}\right|_{j} \\
& <\left|\alpha_{\left(r_{j}\right)}\right|_{j}+\left|\alpha_{\left(r_{j}+1\right)}\right|_{j}+\cdots+\left|\alpha_{(k+1)}\right|_{j}
\end{aligned}
$$

$(j=1, \cdots, \nu)$, define $\beta_{(i)}=\left(\beta_{(i), 1}, \cdots, \beta_{(i), n}\right) \in \boldsymbol{Z}_{+}^{n}(i=1, \cdots, k)$ by 


$$
\beta_{(i), h}= \begin{cases}\alpha_{(i), h}, & \text { if } h \in K_{j} \text { and } \quad r_{j}+1 \leqq i \leqq k, \\ 0, & \text { if otherwise }\end{cases}
$$

$(i=1, \cdots, k$ and $h=1, \cdots, n)$, and put $\beta\left(J_{k}\right)=\left(\beta_{(1)}, \cdots, \beta_{(k)}\right)$. Then, we can see that this $\beta\left(J_{k}\right)$ satisfies the conditions $\beta\left(J_{k}\right) \in \mathscr{M}\left(J_{k}\right)$ and (i) (iii) (as proved below). Note that by (6.23) we have

$$
\left|\beta_{(i)}\right|_{j}= \begin{cases}0, & \text { when } 1 \leqq i \leqq r_{j}, \\ \left|\alpha_{(i)}\right|_{j}, & \text { when } r_{j}+1 \leqq i \leqq k\end{cases}
$$

$(i=1, \cdots, k$ and $j=1, \cdots, \nu)$.

Let us first show the condition $\beta\left(J_{k}\right) \in \mathscr{M}\left(J_{k}\right)$. Since $(0, \cdots, 0) \leqq \beta_{(i)} \leqq \alpha_{(i)}$ $(i=1, \cdots, k)$ is clear from (6.23), what we must prove is the following:

$$
\left\langle\kappa, \beta_{(1)}+\cdots+\beta_{(i)}\right\rangle \leqq l_{1}+\cdots+l_{i} \quad(i=1, \cdots, k) .
$$

Recall that by the definition of $\left\{K_{1}, \cdots, K_{\nu}\right\}$ we can choose $\kappa\left(K_{j}\right)>0(j=1$, $\cdots, \nu)$ such that $\kappa\left(K_{j}\right)=\kappa_{p}$ for any $p \in K_{j}$. Define $\psi_{j}^{(i)}(i=1, \cdots, k$ and $j=1$, $\cdots, \nu)$ by

$$
\psi_{j}^{(i)}= \begin{cases}\kappa\left(K_{j}\right)\left(\left|z_{(1)}\right|_{j}+\cdots+\left|z_{(i)}\right|_{j}\right), & \text { when } 1 \leqq i \leqq r_{j}, \\ \kappa\left(K_{j}\right)\left(\left|z_{(1)}\right|_{j}+\cdots+\left|z_{(i)}\right|_{j}\right) & \\ -\kappa\left(K_{j}\right)\left(\left|\alpha_{\left(r_{j}+1\right)}\right|_{j}+\cdots+\left|\alpha_{(i)}\right|_{j}\right), & \text { when } r_{j}+1 \leqq i \leqq k,\end{cases}
$$

Then, by (6.24), (6.26) and the condition $\left\langle\kappa, z_{(i)}\right\rangle \leqq l_{i}(i=1, \cdots, k)$ we have

$$
\begin{aligned}
& l_{1}+\cdots+l_{i}-\left\langle\kappa, \beta_{(1)}+\cdots+\beta_{(i)}\right\rangle \\
& \quad=l_{1}+\cdots+l_{i}-\left\langle\kappa, z_{(1)}+\cdots+z_{(i)}\right\rangle+\psi_{1}^{(i)}+\cdots+\psi_{\nu}^{(i)} \\
& \quad \geqq \psi_{1}^{(i)}+\cdots+\psi_{\nu}^{(i)}
\end{aligned}
$$

$(i=1, \cdots, k)$. Moreover, we can see the following:

$$
\psi_{j}^{(i)} \geqq 0 \quad(i=1, \cdots, k \text { and } j=1, \cdots, \nu) .
$$

In fact, this is verified as follows: when $1 \leqq i \leqq r_{j}, \psi_{j}^{(i)} \geqq 0$ is clear from (6.26); when $r_{j}+1 \leqq i \leqq k, \psi_{j}^{(i)} \geqq 0$ is verified by $(6.22)$ and

$$
\begin{aligned}
\psi_{j}^{(i)}= & \kappa\left(K_{j}\right)\left[\left(\left|z_{(1)}\right|_{j}+\cdots+\left|z_{(k)}\right|_{j}\right)-\left(\left|\alpha_{\left(r_{j}+1\right)}\right|_{j}+\cdots+\left|\alpha_{(k)}\right|_{j}\right)\right] \\
& +\kappa\left(K_{j}\right)\left[\left(\left|\alpha_{(i+1)}\right|_{j}-\left|z_{(i+1)}\right|_{j}\right)+\cdots+\left(\left|\alpha_{(k)}\right|_{j}-\left|z_{(k)}\right|_{j}\right)\right] .
\end{aligned}
$$

Hence, by (6.27) and (6.28) we obtain (6.25). Thus, the condition $\beta\left(J_{k}\right) \in \mathscr{M}\left(J_{k}\right)$ is proved.

Let us next show the conditions (i) and (ii). Note that by (6.22), (6.23) and (6.24) we have 


$$
\begin{aligned}
& \left(\left|\alpha_{(1)}\right|-\left|\beta_{(1)}\right|\right)+\cdots+\left(\left|\alpha_{(k)}\right|-\left|\beta_{(k)}\right|\right) \\
& =\left(\left|\alpha_{(1)}\right|-\left|z_{(1)}\right|\right)+\cdots+\left(\left|\alpha_{(k)}\right|-\left|z_{(k)}\right|\right) \\
& \quad+\sum_{j=1}^{\nu}\left[\left(\left|z_{(1)}\right|_{j}+\cdots+\left|z_{(k)}\right|_{j}\right)-\left(\left|\alpha_{\left(r_{j}+1\right)}\right|_{j}+\cdots+\left|\alpha_{(k)}\right|_{j}\right)\right] \\
& \geqq\left(\left|\alpha_{(1)}\right|-\left|z_{(1)}\right|\right)+\cdots+\left(\left|\alpha_{(k)}\right|-\left|z_{(k)}\right|\right) .
\end{aligned}
$$

Therefore, by putting

$$
c_{1}=\min \{|\alpha|-|z(j, \alpha)| ;(j, \alpha) \in \mathscr{J}\} \quad(>0)
$$

we can obtain (i). Note that by (6.20) we have

$$
s\left(\left|\alpha_{(i)}\right|-\left|\beta_{(i)}\right|\right) \leqq\left(m-j_{i}-\left|\beta_{(i)}\right|\right)+(s-1)\left(\left|z_{(i)}\right|-\left|\beta_{(i)}\right|\right)
$$

$(i=1, \cdots, k)$. Therefore, by (6.22), (6.23), (6.24) and (6.29) we have

$$
\begin{aligned}
& s\left[\left(\left|\alpha_{(1)}\right|-\left|\beta_{(1)}\right|\right)+\cdots+\left(\left|\alpha_{(k)}\right|-\mid \beta_{(k)}\right)\right] \\
& \quad \leqq \\
& \quad\left(m-j_{1}-\left|\beta_{(1)}\right|\right)+\cdots+\left(m-j_{k}-\left|\beta_{(k)}\right|\right) \\
& \quad+(s-1) \sum_{j=1}^{\nu}\left[\left(\left|z_{(1)}\right|_{j}+\cdots+\left|z_{(k)}\right|_{j}\right)-\left(\left|\alpha_{\left(r_{j+1}\right)}\right|_{j}+\cdots+\left|\alpha_{(k)}\right|_{j}\right)\right] \\
& \quad \leqq \\
& \quad\left(m-j_{1}-\left|\beta_{(1)}\right|\right)+\cdots+\left(m-j_{k}-\left|\beta_{(k)}\right|\right)+(s-1) \sum_{j=1}^{\nu}\left|\alpha_{\left(r_{j}\right)}\right|_{j} .
\end{aligned}
$$

Hence, by putting $c_{2}=(s-1) m \nu(>0)$ we can obtain (ii).

Let us lastly show the condition (iii). Put $\mathscr{J}_{(\mu)}, \mathscr{J}^{(\mu)}, \varepsilon_{(\mu)}, \varepsilon^{(\mu)}(\mu=1, \cdots, \nu)$ as follows:

$$
\begin{aligned}
\mathscr{J}_{(\mu)} & =\left\{(j, \alpha) \in \mathscr{J} ;|z(j, \alpha)|_{\mu}>0\right\}, \\
\mathscr{J}^{(\mu)} & =\left\{(j, \alpha) \in \mathscr{J} ;|\alpha|_{\mu}>|z(j, \alpha)|_{\mu}\right\}, \\
\varepsilon_{(\mu)} & =\min \left\{|z(j, \alpha)|_{\mu} ;(j, \alpha) \in \mathscr{J}_{(\mu)}\right\} \quad(>0), \\
\varepsilon^{(\mu)} & =\min \left\{|\alpha|_{\mu}-|z(j, \alpha)|_{\mu} ;(j, \alpha) \in \mathscr{J}^{(\mu)}\right\} \quad(>0) .
\end{aligned}
$$

Put $I=\{1, \cdots, k\}$, and put $J_{(j)}, J^{(j)}(j=1, \cdots, \nu)$ as follows:

$$
\begin{aligned}
& J_{(j)}=\left\{i \in I ;\left|z_{(i)}\right|_{j}>0 \text { and } 1 \leqq i \leqq r_{j}\right\}, \\
& J^{(j)}=\left\{i \in I ;\left|\alpha_{(i)}\right|_{j}-\left|z_{(i)}\right|_{j}>0 \text { and } r_{j}+1 \leqq i \leqq k\right\} .
\end{aligned}
$$

Then, we can see the following conditions (iii-1) (iii-3):

(iii-1) $\quad\left(J_{(1)} \cup J^{(1)}\right) \cup \cdots \cup\left(J_{(\nu)} \cup J^{(\nu)}\right)=I(=\{1, \cdots, k\})$.

(iii-2) If $J_{(j)}=\left\{h_{1}, h_{2}, \cdots, h_{p}\right\}$ and $h_{1}<h_{2}<\cdots<h_{p}$, we have

$$
\psi_{j}^{\left(h_{d}\right)} \geqq \kappa\left(K_{i}\right) \varepsilon_{(j)} d \quad \text { for } d=1,2, \cdots, p .
$$

(iii-3) If $J^{(j)}=\left\{h_{1}, h_{2}, \cdots, h_{p}\right\}$ and $h_{1}<h_{2}<\cdots<h_{p}$, we have 


$$
\psi_{j}^{\left(h_{d j}\right)} \geqq \kappa\left(K_{j}\right) \varepsilon^{(j)}(p-d) \quad \text { for } d=1,2, \cdots, p \text {. }
$$

In fact, these are verified as follows. (iii-1) is clear from (6.21). (iii-2) is verified by the following: by $(6.26)$ we have

$$
\begin{aligned}
\psi_{j}^{\left(h_{d}\right)}= & \kappa\left(K_{j}\right)\left(\left|z_{(1)}\right|_{j}+\cdots+\left|z_{\left(h_{d}\right)}\right|_{j}\right) \\
& \geqq \kappa\left(K_{j}\right)\left(\left|z_{\left(h_{1}\right)}\right|_{j}+\left|z_{\left(h_{2}\right)}\right|_{j}+\cdots+\left|z_{\left(h_{d}\right)}\right|_{j}\right) \\
& \geqq \kappa\left(K_{j}\right) \varepsilon_{(j)} d .
\end{aligned}
$$

(iii-3) is verified by the following: by (6.22) and (6.26) we have

$$
\begin{aligned}
\psi_{j}^{\left(h_{d}\right)}= & \kappa\left(K_{j}\right)\left[\left(\left|z_{(1)}\right|_{j}+\cdots+\left|z_{(k)}\right|_{j}\right)-\left(\left|\alpha_{\left(r_{j+1}\right)}\right|_{j}+\cdots+\left|\alpha_{(k)}\right|_{j}\right)\right] \\
& +\kappa\left(K_{j}\right)\left[\left(\left|\alpha_{\left(h_{d}+1\right)}\right|_{j}-\left|z_{\left(h_{d}+1\right)}\right|_{j}\right)+\cdots+\left(\left|\alpha_{(k)}\right|_{j}-\left|z_{(k)}\right|_{j}\right)\right] \\
\geqq & \kappa\left(K_{j}\right)\left[\left(\left|\alpha_{\left(h_{d+1}\right)}\right|_{j}-\left|z_{\left(h_{d+1}\right)}\right|_{j}\right)+\left(\left|\alpha_{\left(h_{d+2}\right)}\right|_{j}-\left|z_{\left(h_{d+2}\right)}\right|_{j}\right)\right. \\
& \left.+\cdots+\left(\left|\alpha_{\left(h_{p}\right)}\right|_{j}-\left|z_{\left(h_{p}\right)}\right|_{j}\right)\right] \\
\geqq & \kappa\left(K_{j}\right) \varepsilon^{(j)}(p-d) .
\end{aligned}
$$

By using (iii-1) ( (iii-3), we can prove (iii) as follows. Put

$$
c_{3}=\min \left\{\kappa\left(K_{j}\right) \varepsilon_{(j)}, \kappa\left(K_{j}\right) \varepsilon^{(j)} ; j=1, \cdots, \nu\right\} \quad(>0),
$$

choose a disjoint family $\left\{I_{(j)}, I^{(j)} ; j=1, \cdots, \nu\right\}$ such that $I_{(j)} \subset J_{(j)}, I^{(j)} \subset J^{(j)}$ and $\left(I_{(1)} \cup I^{(1)}\right) \cup \cdots \cup\left(I_{(\nu)} \cup I^{(\nu)}\right)=I$, and define $N_{1}, \cdots, N_{k} \in Z_{+}$as follows: if $I_{(j)}=\left\{i_{1}, i_{2}, \cdots, i_{q}\right\}$ and $i_{1}<i_{2}<\cdots<i_{q}$, we put $N_{i_{d}}=(d-1)(d=1,2, \cdots, q)$; if $I^{(i)}=\left\{i_{1}, i_{2}, \cdots, i_{q}\right\}$ and $i_{1}<i_{2}<\cdots<i_{q}$, we put $N_{i_{d}}=(q-d)(d=1,2, \cdots, q)$. Then, by (6.27), (6.28), (iii-2) and (iii-3) we obtain

$$
\begin{aligned}
l_{1}+\cdots+l_{i}-\left\langle\kappa, \beta_{(1)}+\cdots+\beta_{(i)}\right\rangle & \geqq \psi_{1}^{(i)}+\cdots+\psi_{\nu}^{(i)} \\
& \geqq c_{3} N_{i}
\end{aligned}
$$

$(i=1, \cdots, k)$. Thus, (iii) is proved.

Q.E.D.

As a consequence of Lemma 8, we obtain

Proof of "(I-1) $\Rightarrow(\mathrm{I}-2)$ " IN Proposition 1. Assume that (C-1) or (C-2) holds. Let $c_{1}>0, c_{2}>0$ and $c_{3}>0$ be as in Lemma 8. Take $J_{k}=\left(\left(j_{1}, \alpha_{(1)}\right), \cdots\right.$, $\left.\left(j_{k}, \alpha_{(k)}\right)\right) \in \mathscr{J}^{k}$, let $\beta\left(J_{k}\right)=\left(\beta_{(1)}, \cdots, \beta_{(k)}\right) \in \mathscr{M}\left(J_{k}\right)$ and $N_{1}, \cdots, N_{k} \in Z_{+}$be the ones chosen for $J_{k}$ in Lemma 8 , and let $a_{i}(i=1, \cdots, k)$ be as in (2.7). Then, by (6.19) we have

$$
\frac{1}{a_{i}} \leqq \frac{1}{a+c_{3} N_{i-1}} \leqq \frac{1}{1+N_{i-1}} \frac{1}{\min \left\{a, c_{3}\right\}} \quad(i=1, \cdots, k)
$$

(where $N_{0}=0$ ), and by (6.30), (iii) in Lemma 8 and (3) in Lemma 5 we have 


$$
\begin{aligned}
& \frac{1}{\left(a_{1}\right)^{m-j_{1}-\left|\beta_{(1)}\right|} \times \cdots \times\left(a_{k}\right)^{m-j_{k}-\left|\beta_{(k)}\right|}} \\
& \leqq A_{1}^{k} \frac{1}{\left(1+N_{0}\right)^{m-j_{1}-\left|\beta_{(1)}\right|} \times \cdots \times\left(1+N_{k-1}\right)^{m-j_{k}-\left|\beta_{(k)}\right|}} \\
& \leqq A_{2}^{k+1} \frac{1}{\left[\left(m-j_{1}-\left|\beta_{(1)}\right|\right)+\cdots+\left(m-j_{k}-\left|\beta_{(k)}\right|\right)\right] !}
\end{aligned}
$$

for some $A_{1}>0$ and $A_{2}>0$ (depending only on $a, c_{3}, m$ and $\nu$ ). Therefore, by (6.31), (ii) in Lemma 8 and (1) in Lemma 5 we have

$$
\frac{\left[\left(\left|\alpha_{(1)}\right|-\left|\beta_{(1)}\right|\right)+\cdots+\left(\left|\alpha_{(k)}\right|-\left|\beta_{(k)}\right|\right)\right] !^{s}}{\left(a_{1}\right)^{m-j_{1}-\left|\beta_{(1)}\right|} \times \cdots \times\left(a_{k}\right)^{m-j_{k-1}\left|\beta_{(k)}\right|}} \leqq B^{k+1}
$$

for some $B>0$ (which is independent of $J_{k}, \beta\left(J_{k}\right)$ and $k$ ). Hence, if we assume that $0<R \leqq 1$, by (2.7), (6.32) and (i) in Lemma 8 we obtain

$$
F_{a}\left(J_{k}, \beta\left(J_{k}\right), C, R, T\right) \leqq H^{k+1} R^{c_{1} k}
$$

for some $H>0$ (which is independent of $J_{k}, \beta\left(J_{k}\right)$ and $k$ ). This leads us to the condition (I-2) in Proposition 1.

Q.E.D.

In the above proof, the following fact is essential: the conditions (i), (ii) in Lemma 8 and (6.32) imply (I-2) (and hence (I-3)). The following lemmas 9 and 10 assert that the converse relation holds.

Lemma 9. There is a $d>0$ which satisfies the following condition: if $J_{k}=\left(\left(j_{1}, \alpha_{(1)}\right), \cdots,\left(j_{k}, \alpha_{(k)}\right)\right) \in \mathscr{J}^{k}$ satisfies $0<l\left(j_{i}, \alpha_{(i)}\right)<\left\langle k, \alpha_{(i)}\right\rangle \quad(i=1, \cdots, k)$, we have

$$
\left(\left|\alpha_{(1)}\right|-\left|\beta_{(1)}\right|\right)+\cdots+\left(\left|\alpha_{(k)}\right|-\left|\beta_{(k)}\right|\right) \geqq d k
$$

for any $\beta\left(J_{k}\right)=\left(\beta_{(1)}, \cdots, \beta_{(k)}\right) \in \mathscr{M}\left(J_{k}\right)$.

Proof. Put

$$
\begin{aligned}
& \mathscr{J}_{0}=\{(j, \alpha) \in \mathscr{J} ; 0<l(j, \alpha)<\langle\kappa, \alpha\rangle\}, \\
& c=\min \left\{\langle\kappa, \alpha\rangle-l(j, \alpha) ;(j, \alpha) \in \mathscr{J}_{0}\right\} \quad(>0), \\
& \kappa^{*}=\max \left\{\kappa_{1}, \cdots, \kappa_{n}\right\} .
\end{aligned}
$$

Take any $\beta\left(J_{k}\right)=\left(\beta_{(1)}, \cdots, \beta_{(k)}\right) \in \mathscr{M}\left(J_{k}\right)$. Then, by (2.6), (6.34) and the condition $\left(j_{i}, \alpha_{(i)}\right) \in \mathscr{J}_{0}(i=1, \cdots, k)$ we have

$$
\begin{aligned}
&\left.\kappa^{*}\left[\left(\left|\alpha_{(1)}\right|-\left|\beta_{(1)}\right|\right)+\cdots+\left(\left|\alpha_{(k)}\right|-\mid \beta_{(k)}\right)\right)\right] \geqq\left\langle\kappa, \alpha_{(1)}-\beta_{(1)}\right\rangle+\cdots+\left\langle\kappa, \alpha_{(k)}-\beta_{(k)}\right\rangle \\
&=\left(\left\langle\kappa, \alpha_{(1)}\right\rangle-l\left(j_{1}, \alpha_{(1)}\right)\right)+\cdots+\left(\left\langle\kappa, \alpha_{(k)}\right\rangle-l\left(j_{k}, \alpha_{(k)}\right)\right) \\
&+\left(l\left(j_{1}, \alpha_{(1)}\right)+\cdots+l\left(j_{k}, \alpha_{(k)}\right)-\left\langle\kappa, \beta_{(1)}+\cdots+\beta_{(k)}\right\rangle\right) \geqq c k .
\end{aligned}
$$


Hence, by putting $d=c / \kappa^{*}$ we obtain (6.33).

Q.E.D.

Lemma 10. Assume the condition (I-3) in Proposition 1. Then, there are $A>0$ and $B>0$ such that for any $J_{k}=\left(\left(j_{1}, \alpha_{(1)}\right), \cdots,\left(j_{k}, \alpha_{(k)}\right)\right) \in \mathscr{J}^{k}$ we can find $\beta\left(J_{k}\right)=\left(\beta_{(1)}, \cdots, \beta_{(k)}\right) \in \mathscr{M}\left(J_{k}\right)$ and $c\left(J_{k}\right) \geqq 0$ which satisfy the following conditions (i) (iii).

(i ) $\frac{\left[\left(\left|\alpha_{(1)}\right|-\left|\beta_{(1)}\right|\right)+\cdots+\left(\left|\alpha_{(k)}\right|-\left|\beta_{(k)}\right|\right)\right] !^{s}}{\left(a_{1}\right)^{m-j_{1}-\left|\beta_{(1)}\right|} \times \cdots \times\left(a_{k}\right)^{m-j_{k}-\left|\beta_{(k)}\right|}} \leqq A B^{k}$

where $a_{1}=a(>0)$ and

$a_{i+1}=l\left(j_{1}, \alpha_{(1)}\right)+\cdots+l\left(j_{i}, \alpha_{(i)}\right)-\left\langle\kappa, \beta_{(1)}+\cdots+\beta_{(i)}\right\rangle \quad(i=1, \cdots, k)$.

(ii) $\frac{c\left(J_{k}\right)+\left(m-j_{1}-\left|\beta_{(1)}\right|\right)+\cdots+\left(m-j_{k}-\left|\beta_{(k)}\right|\right)}{\left(\left|\alpha_{(1)}\right|-\left|\beta_{(1)}\right|\right)+\cdots+\left(\left|\alpha_{(k)}\right|-\left|\beta_{(k)}\right|\right)} \geqq s$.

(iii) $\max _{J_{k} \in \mathscr{g}^{k}}\left\{c\left(J_{k}\right)\right\}=o(k) \quad($ as $k \rightarrow \infty)$.

Proof. Assume the condition (I-3) in Proposition 1. Then,

$$
M_{a}\left(\left\{\beta\left(J_{k}\right)\right\}, C, R_{0}, T_{0}\right)<+\infty
$$

for some $\beta\left(J_{k}\right)=\left(\beta_{(1)}, \cdots, \beta_{(k)}\right) \in \mathscr{M}\left(J_{k}\right)$ (where $J_{k}=\left(\left(j_{1}, \alpha_{(1)}\right), \cdots,\left(j_{k}, \alpha_{(k)}\right)\right) \in \mathscr{J}^{k}$, $k=1,2, \cdots), C>0, R_{0}>0$ and $T_{0}>0$. Therefore, by (2.7) and (2.8) we have

$$
\frac{\left[\left(\left|\alpha_{(1)}\right|-\left|\beta_{(1)}\right|\right)+\cdots+\left(\left|\alpha_{(k)}\right|-\left|\beta_{(k)}\right|\right)\right] !^{s}}{\left(a_{1}\right)^{m-j_{1}-\left|\beta_{(1)}\right|} \times \cdots \times\left(a_{k}\right)^{m-j_{k}-\left|\beta_{(k)}\right|}} \leqq A B^{k}
$$

for some $A>0$ and $B>0$ (which are independent of $J_{k}, \beta\left(J_{k}\right)$ and $k$ ); this is (i). Put $l=\max \{l(j, \alpha) ;(j, \alpha) \in \mathscr{J}\}$. Since

$$
\frac{1}{a_{i}} \geqq \frac{1}{a+l(i-1)} \geqq \frac{1}{i} \frac{1}{\max \{a, l\}} \quad(i=1, \cdots, k),
$$

by (6.35) and (6.36) we have

$$
\frac{\left[\left(\left|\alpha_{(1)}\right|-\left|\beta_{(1)}\right|\right)+\cdots+\left(\left|\alpha_{(k)}\right|-\left|\beta_{(k)}\right|\right)\right] !^{s}}{(1)^{m-j_{1}-\left|\beta_{(1)}\right|} \times \cdots \times(k)^{m-j_{k}-\left|\beta_{(k)}\right|}} \leqq A B_{1}^{k}
$$

for $B_{1}=B(\max \{1, a, l\})^{m}$. Hence, by applying (3) in Lemma 5 and Stirling's formula to (6.37) we obtain

$$
\frac{\left.\left[s\left(\left|\alpha_{(1)}\right|-\left|\beta_{(1)}\right|\right)+\cdots+s\left(\left|\alpha_{(k)}\right|-\mid \beta_{(k)}\right)\right)\right] !}{\left[\left(m-j_{1}-\left|\beta_{(1)}\right|\right)+\cdots+\left(m-j_{k}-\left|\beta_{(k)}\right|\right)\right] !} \leqq C H^{k}
$$

for some $C>0$ and $H>0$ (which are independent of $J_{k}, \beta\left(J_{k}\right)$ and $k$ ).

Here, we put $b\left(J_{k}\right)$ and $c\left(J_{k}\right)$ as follows: 


$$
\begin{aligned}
b\left(J_{k}\right)= & {\left[s\left(\left|\alpha_{(1)}\right|-\left|\beta_{(1)}\right|\right)+\cdots+s\left(\left|\alpha_{(k)}\right|-\left|\beta_{(k)}\right|\right)\right] } \\
& -\left[\left(m-j_{1}-\left|\beta_{(1)}\right|\right)+\cdots+\left(m-j_{k}-\left|\beta_{(k)}\right|\right)\right], \\
c\left(J_{k}\right)= & \max \left\{0, b\left(J_{k}\right)\right\} \quad(\geqq 0) .
\end{aligned}
$$

Then, we have $c\left(J_{k}\right) \geqq b\left(J_{k}\right)$ and therefore by (6.39) we obtain (ii). Moreover, we can see the following: there are $C_{1}>0$ and $C_{2}>0$ such that

$$
\log \Gamma\left(1+c\left(J_{k}\right)\right) \leqq C_{1} k+C_{2}
$$

holds for any $J_{k}$ and $k$. In fact, this is verified as follows: by (6.38) and (6.39) we have

$$
\frac{\left[b\left(J_{k}\right)+\left(m-j_{1}-\left|\beta_{(1)}\right|\right)+\cdots+\left(m-j_{k}-\left|\beta_{(k)}\right|\right)\right] !}{\left[\left(m-j_{1}-\left|\beta_{(1)}\right|\right)+\cdots+\left(m-j_{k}-\left|\beta_{(k)}\right|\right)\right] !} \leqq C H^{k}
$$

and therefore by (1) in Lemma 5 we obtain

$$
\left[c\left(J_{k}\right)\right] ! \leqq C_{0} H_{0}^{k}
$$

(which implies (6.40)) for $C_{0}=\max \{1,2 C\}$ and $H_{0}=\max \left\{1,2^{m s} H\right\}$.

Hence, by using (6.40) we can verify the condition (iii) as follows. Assume that $\left\{c\left(J_{k}\right)\right\}$ does not satisfy (iii). Then, there are $\varepsilon>0, k_{1}<k_{2}<\ldots$ and $J^{(i)} \in \mathscr{J}^{k_{i}}(i=1,2, \cdots)$ such that $c\left(J^{(i)}\right) \geqq \varepsilon k_{i}(i=1,2, \cdots)$ and $k_{i} \rightarrow \infty$ (as $i \rightarrow \infty)$; therefore, by (6.40) we have

$$
\log \Gamma\left(1+\varepsilon k_{i}\right) \leqq C_{1} k_{i}+C_{2} \quad(i=1,2, \cdots) .
$$

This contradicts the condition $k_{i} \rightarrow \infty$ (as $i \rightarrow \infty$ ). Thus, (iii) is also verified.

Q.E.D.

Corollary to Lemmas 9 and 10. Assume the condition (I-3) in Proposition 1. Let $(j, \alpha) \in \mathscr{J}$. Then we have the following results.

(1) If $0<l(j, \alpha)<\langle\kappa, \alpha\rangle$ holds, there are $d>0, A>0, B>0, z_{(k)} \in \mathscr{Z}(j, \alpha)$ $(k=1,2, \cdots)$ and $c_{k} \geqq 0(k=1,2, \cdots)$ which satisfy the following conditions (i) (iv).

(i ) $|\alpha|-\left|z_{(k)}\right| \geqq d \quad(k=1,2, \cdots)$.

(ii) For any $k$, we can find $a\left(\beta_{(1)}, \cdots, \beta_{(k)}\right) \in \boldsymbol{Z}_{+}^{n} \times \cdots \times \boldsymbol{Z}_{+}^{n}$ such that $(0, \cdots, 0) \leqq \beta_{(i)} \leqq \alpha(i=1, \cdots, k), \beta_{(1)}+\cdots+\beta_{(k)}=k z_{(k)},\left\langle\kappa, \beta_{(1)}+\cdots+\beta_{(i)}\right\rangle \leqq$ $i l(j, \alpha)(i=1, \cdots, k)$ and

$$
\frac{\left[k\left(|\alpha|-\left|z_{(k)}\right|\right)\right] !^{s}}{\left(a_{1}\right)^{m-j_{1}-\left|\beta_{(1)}\right|} \times \cdots \times\left(a_{k}\right)^{m-j_{k}-\left|\beta_{(k)}\right|}} \leqq A B^{k},
$$

where $a_{1}=a$ and $a_{i+1}=a+i l(j, \alpha)-\left\langle\kappa, \beta_{(1)}+\cdots+\beta_{(i)}\right\rangle(i=1, \cdots, k)$.

(iii) $s \leqq c_{k}+\frac{m-j-\left|z_{(k)}\right|}{|\alpha|-\left|z_{(k)}\right|} \quad(k=1,2, \cdots)$. 
(iv) $c_{k}=o(1) \quad($ as $k \rightarrow \infty)$.

(2) Moreover, if $s=\sigma_{j, \alpha} /\left(\sigma_{j, \alpha}-1\right)$ and $l(j, \alpha) \in S_{k}(\alpha)$ hold, the $z_{(k)}(k=$ $1,2, \ldots)$ in (1) satisfy also the following condition:

$$
z_{(k)} \rightarrow z^{*} \quad(\text { as } k \rightarrow \infty),
$$

where $z^{*}$ is the one in Corollary to (3) in Lemma 7.

Proof. Assume the condition (I-3) in Proposition 1. Let $(j, \alpha) \in \mathscr{J}$ and assume that $0<l(j, \alpha)<\langle\kappa, \alpha\rangle$ holds. Let $d>0$ be as in Lemma 9 , let $A>0$, $B>0$ be as in Lemma 10 , put $J_{k}^{0}=((j, \alpha), \cdots,(j, \alpha)) \in \mathscr{J}^{k}$, let $\beta\left(J_{k}^{0}\right)=\left(\beta_{(1)}, \cdots\right.$, $\left.\beta_{(k)}\right) \in \mathscr{M}\left(J_{k}^{0}\right)$ be the one chosen for $J_{k}^{0}$ in Lemma 10, and define $z_{(k)}, c_{k}$ by

$$
\begin{aligned}
z_{(k)} & =\frac{1}{k}\left(\beta_{(1)}+\cdots+\beta_{(k)}\right), \\
c_{k} & =\frac{c\left(J_{k}^{0}\right)}{d k}
\end{aligned}
$$

$(k=1,2, \cdots)$. Then $(i)$ is verified by Lemma 9 and (ii) (iv) are verified by Lemma 10. Hence, we obtain (1).

Next, let us prove (2). Assume that $s=\sigma_{1, \alpha} /\left(\sigma_{j, \alpha}-1\right)$ and $l(j, \alpha) \in S_{k}(\alpha)$ hold. Let $\nu \in \widetilde{S}_{n}, q \in\{1, \cdots, n-1\}$ and $z^{*}=\left(z_{1}^{*}, \cdots, z_{n}^{*}\right) \in Z_{+}^{n}$ be as in Corollary to (3) in Lemma 7. Put $z_{(k)}=\left(z_{(k), 1}, \cdots, z_{(k), n}\right), a=\max \left\{\kappa_{\nu(1)}, \cdots, \kappa_{\nu(q)}\right\}$ and $b=\min \left\{\kappa_{\nu(q+1)}, \cdots, \kappa_{\nu(n)}\right\}$. Then, we have $z_{\nu(i)}^{*}=\alpha_{\nu(i)}$ for $1 \leqq i \leqq q, z_{\nu(i)}^{*}=0$ for $q+1 \leqq i \leqq n,\left\langle\kappa, z^{*}\right\rangle=l(j, \alpha) \geqq\left\langle\kappa, z_{(k)}\right\rangle,(0, \cdots, 0) \leqq z_{(k)} \leqq \alpha,(1 / a) \kappa_{\nu(i)} \leqq 1$ for $1 \leqq i$ $\leqq q,(1 / b) \kappa_{\nu(i)} \geqq 1$ for $q+1 \leqq i \leqq n, 0<a<b$ and

$$
\begin{aligned}
\left|z^{*}\right|-\left|z_{(k)}\right|= & \left(\alpha_{\nu(1)}-z_{(k), \nu(1)}\right)+\cdots+\left(\alpha_{\nu(q)}-z_{(k), \nu(q)}\right) \\
& -\left(z_{(k), \nu(q+1)}+\cdots+z_{(k), \nu(n)}\right) \\
\geqq & \frac{1}{a}\left[\kappa_{\nu(1)}\left(\alpha_{\nu(1)}-z_{(k), \nu(1)}\right)+\cdots+\kappa_{\nu(q)}\left(\alpha_{\nu(q)}-z_{(k), \nu(q)}\right)\right] \\
& -\frac{1}{b}\left(\kappa_{\nu(q+1)} z_{(k), \nu(q+1)}+\cdots+\kappa_{\nu(n)} z_{(k), \nu(n)}\right) \\
= & \frac{1}{a}\left(\left\langle\kappa, z^{*}\right\rangle-\left\langle\kappa, z_{(k)}\right\rangle\right) \\
& +\left(\frac{1}{a}-\frac{1}{b}\right)\left(\kappa_{\nu(q+1)} z_{(k), \nu(q+1)}+\cdots+\kappa_{\nu(n)} z_{(k), \nu(n)}\right) \\
\geqq & \left(\frac{b}{a}-1\right)\left(z_{(k), \nu(q+1)}+\cdots+z_{(k, \nu(n)}\right) .
\end{aligned}
$$

On the other hand, we know from (6.18), (iii), (iv) and the condition $\left|z^{*}\right| \geqq$ $\left|\boldsymbol{z}_{(k)}\right|$ (by (6.42)) that 


$$
\begin{aligned}
\left|z^{*}\right|-\left|z_{(k)}\right| & =\frac{\left(|\alpha|-\left|z^{*}\right|\right)\left(|\alpha|-\left|z_{(k)}\right|\right)}{(m-j-|\alpha|)}\left(\frac{m-j-\left|z^{*}\right|}{|\alpha|-\left|z^{*}\right|}-\frac{m-j-\left|z_{(k)}\right|}{|\alpha|-\left|z_{(k)}\right|}\right) \\
& =\frac{\left(|\alpha|-\left|z^{*}\right|\right)\left(|\alpha|-\left|z_{(k)}\right|\right)}{(m-j-|\alpha|)}\left(s-\frac{m-j-\left|z_{(k)}\right|}{|\alpha|-\left|z_{(k)}\right|}\right) \\
& =o(1) \quad(\text { as } k \rightarrow \infty) .
\end{aligned}
$$

Therefore, by (6.42) and (6.43) we have

$$
z_{(k), \nu(q+1)}+\cdots+z_{(k), \nu(n)}=o(1) \quad(\text { as } k \rightarrow \infty),
$$

and by (6.43) and (6.44) we have

$$
\begin{aligned}
& \left(\alpha_{\nu(1)}-z_{(k), \nu(1)}\right)+\cdots+\left(\alpha_{\nu(q)}-z_{(k), \nu(q)}\right) \\
& \quad=\left|z^{*}\right|-\left|z_{(k)}\right|+\left(z_{(k), \nu(q+1)}+\cdots+z_{(k), \nu(n)}\right) \\
& \quad=o(1) \quad(\text { as } k \rightarrow \infty) .
\end{aligned}
$$

Hence, we obtain $z_{(k)} \rightarrow z^{*}$ (as $k \rightarrow \infty$ ), since (6.44) and (6.45) imply

$$
z_{(k), \nu(i)} \longrightarrow \begin{cases}\alpha_{\nu(i)}, & \text { when } 1 \leqq i \leqq q, \\ 0, & \text { when } q+1 \leqq i \leqq n\end{cases}
$$

(as $k \rightarrow \infty)$.

Q.E.D.

As a consequence of the above corollary and the condition (4) in Lemma 7, we obtain

Proof of "(I-3) $\Rightarrow(\mathrm{I}-1)$ " In Propositron 1. Assume the condition (I-3) in Proposition 1. Put (C-3) and (C-4) as follows:

(C-3) $s=\sigma /(\sigma-1)$ and $\Delta_{P} \cap S_{P} \neq \varnothing$,

(C-4) $s>\sigma /(\sigma-1)$.

Then, to have (I-1) in Proposition 1 it is sufficient to prove the following: if we assume that (C-3) or (C-4) holds, we can obtain a contradiction. Let us show this.

First, let us discuss the case (C-4). Assume that (C-4) holds; that is, $s>\sigma_{j, \alpha} /\left(\sigma_{j, \alpha}-1\right)$ holds for some $(j, \alpha) \in \mathscr{J}$. Take such a $(j, \alpha) \in \mathscr{J}$ and fix it. Then, we have $\sigma_{j, \alpha}>1$ and hence $0<l(j, \alpha)<\langle\kappa, \alpha\rangle$ (by (1) in Lemma 6). Therefore, by (1) in Corollary to Lemmas 9 and 10 we can find $z_{(k)} \in \mathscr{Z}(j, \alpha)$ $(k=1,2, \cdots)$ such that

$$
s \leqq o(1)+\frac{m-j-\left|z_{(k)}\right|}{|\alpha|-\left|z_{(k)}\right|}
$$

(as $k \rightarrow \infty)$. Thus, by choosing $s_{0}>0$ so that $s>s_{0}>\sigma_{j, \alpha} /\left(\sigma_{j, \alpha}-1\right)$ and by 
taking $k$ sufficiently large we obtain

$$
\sigma_{j, \alpha} /\left(\sigma_{j, \alpha}-1\right)<s_{0}<\frac{m-j-\left|z_{(k)}\right|}{|\alpha|-\left|z_{(k)}\right|},
$$

which contradicts the condition (4) in Lemma 7.

Next, let us discuss the case (C-3). Assume that (C-3) holds; that is, $s=\sigma_{j, \alpha} /\left(\sigma_{j, \alpha}-1\right)$ and $l(j, \alpha) \in S_{k}(\alpha)$ hold for some $(j, \alpha) \in \mathscr{J}$. Take such a $(j, \alpha) \in \mathscr{J}$ and fix it. Let $A>0, B>0$ and $z_{(k)} \in \mathscr{Z}(j, \alpha)(k=1,2, \ldots)$ be as in Corollary to Lemmas 9 and 10 , and let $z^{*} \in Z_{+}^{n}$ be the one in Corollary to (3) in Lemma 7. Then, we can see that

$$
\frac{\left[k\left(|\alpha|-\left|z_{(k)}\right|\right)\right] !^{s}}{\left(a+k\left|\left\langle\kappa, z^{*}-z_{(k)}\right\rangle\right|\right)^{k\left(m-j-\left|z_{(k)}\right|\right)}} \leqq A B^{k}
$$

holds for any $k$. In fact, this is verified as follows. Note that $\left\langle\kappa, z^{*}\right\rangle=l(j, \alpha)$, and let $\left(\beta_{(1)}, \cdots, \beta_{(k)}\right) \in \boldsymbol{Z}_{+}^{n} \times \cdots \times \boldsymbol{Z}_{+}^{n}$ be the one in (ii) in Corollary to Lemmas 9 and 10. Then, we have $(0, \cdots, 0) \leqq \beta_{(i)} \leqq \alpha(i=1, \cdots, k), k z_{(k)}=\left(\beta_{(1)}+\cdots\right.$ $\left.+\beta_{(k)}\right)$,

$$
\left(m-j-\left|\beta_{(1)}\right|\right)+\cdots+\left(m-j-\left|\beta_{(k)}\right|\right)=k\left(m-j-\left|z_{(k)}\right|\right),
$$

and by (6.17) we have

$$
\begin{aligned}
0 & \leqq i l(j, \alpha)-\left\langle\kappa, \beta_{(1)}+\cdots+\beta_{(i)}\right\rangle=\sum_{h=1}^{i}\left\langle\kappa, z^{*}-\beta_{(h)}\right\rangle \\
& \leqq \sum_{h=1}^{i}\left|\left\langle\kappa, z^{*}-\beta_{(h)}\right\rangle\right| \\
& \leqq \sum_{h=1}^{k}\left|\left\langle\kappa, z^{*}-\beta_{(h)}\right\rangle\right|=k\left|\left\langle\kappa, z^{*}-z_{(k)}\right\rangle\right|
\end{aligned}
$$

$(i=1, \cdots, k)$. Hence, by (6.41), (6.47) and (6.48) we obtain (6.46).

Further, we can also see that

$$
\frac{m-j-\left|z_{(k)}\right|}{|\alpha|-\left|z_{(k)}\right|} \leqq s \quad \text { for any } k \in N
$$

In fact, if otherwise, we can find a $k \in N$ such that

$$
\frac{m-j-\left|z_{(k)}\right|}{|\alpha|-\left|z_{(k)}\right|}>s=\sigma_{j, \alpha} /\left(\sigma_{j, \alpha}-1\right),
$$

which contradicts the condition (4) in Lemma 7.

Hence, by (6.46), (6.49) and (2) in Corollary to Lemmas 9 and 10 we have the following: for any $\varepsilon>0$ there is a $C_{\varepsilon}>0$ such that 


$$
\begin{aligned}
{\left[k\left(|\alpha|-\left|z_{(k)}\right|\right)\right] !^{!} } & \leqq A B^{k}\left(\varepsilon k+C_{\varepsilon}\right)^{k\left(m-j-\left|z_{(k)}\right|\right)} \\
& \leqq A B^{k}\left(\varepsilon k+C_{\varepsilon}\right)^{s k\left(|\alpha|-\left|z_{(k)}\right|\right)}
\end{aligned}
$$

holds for any $k$. In addition, by applying Stirling's formula to the left hand side of (6.50) and by using the condition $d \leqq\left(|\alpha|-\left|z_{(k)}\right|\right) \leqq m(k=1,2, \cdots)$ we have

$$
k \leqq H\left(\varepsilon k+C_{\varepsilon}\right) \quad(k=1,2, \cdots)
$$

for some $H>0$ (which is independent of $\varepsilon$ and $k$ ). Thus, by choosing $\varepsilon>0$ so that $\varepsilon<1 / H$ and by letting $k \rightarrow \infty$ in (6.51) we can obtain a contradiction.

Q.E.D.

Lastly, let us show "(II-3) $\Rightarrow$ (II-1)" in Proposition 1 by the argument similar to that above. Note that the difference between (II-3) and (I-3) lies in whether we can take $R>0$ sufficiently large or not, and that $T_{0}$ depends on $R$ in (II-3).

Proof of "(II-3) $\Rightarrow$ (II-1)" IN Proposition 1. Our purpose is to prove the following: if we assume that (II-3) in Proposition 1 and $s \geqq \sigma /(\sigma-1)$ hold, we can obtain a contradiction.

Assume the condition (II-3) in Proposition 1. Let $R>1$ be sufficiently large. Then, we have

$$
M_{a}\left(\left\{\beta\left(J_{k}\right)\right\}, C, R, T_{0}\right)<+\infty
$$

for some $T_{0}>0$ and $\beta\left(J_{k}\right)=\left(\beta_{(1)}, \cdots, \beta_{(k)}\right) \in \mathscr{M}\left(J_{k}\right)$ (where $J_{k}=\left(\left(j_{1}, \alpha_{(1)}\right), \cdots\right.$, $\left.\left.\left(j_{k}, \alpha_{(k)}\right)\right) \in \mathscr{J}^{k}, k=1,2, \cdots\right)$. Therefore, by (2.7), (2.8) and (6.36) we have

$$
\begin{aligned}
& \frac{\left[\left(\left|\alpha_{(1)}\right|-\left|\beta_{(1)}\right|\right)+\cdots+\left(\left|\alpha_{(k)}\right|-\left|\beta_{(k}\right|\right)\right] !^{s}}{(1)^{m-j_{1}-\left|\beta_{(1)}\right|} \times \cdots \times(k)^{m-j_{k}-\left|\beta_{(k)}\right|}} \\
& \leqq A B^{k}\left(\frac{1}{R}\right)^{\left(\left|\alpha_{(1)}\right|-\left|\beta_{(1)}\right|\right)+\cdots+\left(\left|\alpha_{(k)}\right|-\left|\beta_{(k)}\right|\right)} \\
& \quad \times\left(\frac{1}{T_{0}}\right)^{l\left(j_{1}, \alpha_{(1)}\right)+\cdots+l\left(j_{k}, \alpha_{(k)}\right)-\left\langle k, \beta_{(1)}+\cdots+\beta_{(k)}\right\rangle}
\end{aligned}
$$

for some $A>0$ and $B>0$ (which are independent of $J_{k}, \beta\left(J_{k}\right), k, R$ and $T_{0}$ ).

Assume that $s \geqq \sigma /(\sigma-1)$ holds, that is, $s \geqq \sigma_{j, \alpha} /\left(\sigma_{j, \alpha}-1\right)$ holds for some $(j, \alpha) \in \mathscr{J}$. Take such a $(j, \alpha) \in \mathscr{J}$ and fix it. Then, by the same argument as in Corollary to Lemmas 9 and 10 we can find $d>0, A_{1}>0, B_{1}>0$ (independent of $k, R$ and $\left.T_{0}\right), z_{(k)} \in \mathscr{Z}(j, \alpha)(k=1,2, \cdots)$ and $c_{k} \geqq 0(k=1,2, \cdots)$ which satisfy the following conditions (i) $\sim(\mathrm{iv})$ :

$$
\text { (i ) }|\alpha|-\left|z_{(k)}\right| \geqq d \quad(k=1,2, \cdots) \text {. }
$$


(ii) $\frac{\left[k\left(|\alpha|-\left|\boldsymbol{z}_{(k, \mid)}\right|\right) !^{s}\right.}{\left[k\left(m-j-\left|\boldsymbol{z}_{(k)}\right|\right)\right] !} \leqq A_{1} B_{1}^{k}\left(\frac{1}{R}\right)^{k\left(|\alpha|-\left|z_{(k)}\right|\right)}\left(\frac{1}{T_{0}}\right)^{k\left(l(j, \alpha)-\left\langle k, \boldsymbol{z}_{(k)}\right\rangle\right)}$

$$
(k=1,2, \cdots) \text {. }
$$

(iii) $s \leqq c_{k}+\frac{m-j-\left|z_{(k)}\right|}{|\alpha|-\left|z_{(k)}\right|} \quad(k=1,2, \cdots)$.

(iv) $c_{k}=o(1) \quad($ as $k \rightarrow \infty)$.

By using these conditions, we can obtain a contradiction as follows.

When $s>\sigma_{j, \alpha} /\left(\sigma_{j, \alpha}-1\right)$, by choosing $s_{0}>0$ so that $s>s_{0}>\sigma_{j, \alpha} /\left(\sigma_{j, \alpha}-1\right)$ and by taking $k$ sufficiently large we have

$$
\sigma_{j, \alpha} /\left(\sigma_{j, \alpha}-1\right)<s_{0}<\frac{m-j-\left|z_{(k)}\right|}{|\alpha|-\left|z_{(k)}\right|}
$$

(by (iii) and (iv)), which contradicts the condition (4) in Lemma 7.

When $s=\sigma_{j, \alpha} /\left(\sigma_{j, \alpha}-1\right)$ and when

$$
s<\frac{m-j-\left|z_{(k)}\right|}{|\alpha|-\left|z_{(k)}\right|} \quad \text { for some } k \in N,
$$

we can find $s_{0}>0$ such that

$$
\sigma_{j, \alpha} /\left(\sigma_{j, \alpha}-1\right)=s<s_{0}<\frac{m-j-\left|z_{(k)}\right|}{|\alpha|-\left|z_{(k)}\right|},
$$

which contradicts the condition (4) in Lemma 7.

When $s=\sigma_{j, \alpha} /\left(\sigma_{j, \alpha}-1\right)$ and when

$$
\frac{m-j-\left|z_{(k)}\right|}{|\alpha|-\left|z_{(k)}\right|} \leqq s \quad \text { for any } k \in N,
$$

by (i), (ii), (6.53) and Stirling's formula we have

$$
1 \leqq H\left(\frac{1}{R}\right)^{d}\left(\frac{1}{T_{0}}\right)^{l(j, \alpha)-\left\langle k, z_{(k)}\right\rangle} \quad(k=1,2, \cdots)
$$

for some $H>0$ (which is independent of $k, R$ and $T_{0}$ ). Since $R$ is sufficiently large, we may assume that $H / R^{d}<1$. Therefore, to have a contradiction from (6.54) it is sufficient to prove the following: there is a subsequence $\left\{z_{\left(k_{i}\right)}\right\}$ of $\left\{\boldsymbol{z}_{(k)}\right\}$ such that

$$
\left\langle\kappa, z_{\left(k_{i}\right)}\right\rangle \longrightarrow l(j, \alpha) \quad\left(\text { as } k_{i} \rightarrow \infty\right) .
$$

Let us show this now. Since $\left\{\boldsymbol{z}_{(k)}\right\}$ is a bounded sequence in $\boldsymbol{R}^{n}$, we can take a convergent subsequence $\left\{\boldsymbol{z}_{\left(k_{i}\right)}\right\}$ of $\left\{\boldsymbol{z}_{(k)}\right\}$. Then, the limit $z_{*} \in \boldsymbol{R}^{n}$ of $\left\{\boldsymbol{z}_{\left(\mathrm{b}_{i}\right)}\right\}$ satisfies $\boldsymbol{z}_{*} \in \mathscr{Z}(j, \alpha)$ (by (i)), 


$$
s=\frac{m-j-\left|z_{*}\right|}{|\alpha|-\left|z_{*}\right|}
$$

(by (6.53), (iii) and (iv)), and $\left\langle\kappa, z_{*}\right\rangle \leqq l(j, \alpha)$. Furthermore, we can see the following: if $\left\langle\kappa, z_{*}\right\rangle\left\langle l(j, \alpha)\right.$ holds, we can find a $w \in \mathscr{Z}(j, \alpha)$ satisfying $\left|z_{*}\right|<$ $|w|$ and this yields

$$
\sigma_{j, \alpha} /\left(\sigma_{j, \alpha}-1\right)=s=\frac{m-j-\left|z_{*}\right|}{|\alpha|-\left|z_{*}\right|}<\frac{m-j-|w|}{|\alpha|-|w|},
$$

which contradicts the condition (4) in Lemma 7. Hence, we obtain $\left\langle\kappa, z_{*}\right\rangle$ $=l(j, \alpha)$ and therefore

$$
\left\langle\kappa, z_{\left(k_{i}\right)}\right\rangle \longrightarrow\left\langle\kappa, z_{*}\right\rangle=l(j, \alpha) \quad\left(\text { as } k_{i} \rightarrow \infty\right) .
$$

Q.E.D.

\section{REFERENCES}

[1] V. Ja. Ivrii, Cauchy problem conditions for hyperbolic operators with characteristic of variable multiplicity for Gevrey classes, Sibirsk. Mat. Ž., 17 (1976), 1256-1270. (English translation, Siberian Math. J., 17 (1976), 921-931.)

[2] J. Leray and Y. Ohya, Systèmes linéaires, hyperboliques non stricts, Colloque CBRM, 1964, 105-144.

[ 3 ] H. Tahara, Singular hyperbolic systems, III. On the Cauchy problem for Fuchsian hyperbolic partial differential equations, J. Fac. Sci. Univ. Tokyo, Sect. IA Math., 27 (1980), 465-507.

[4] H. Tahara, Singular hyperbolic systems, V. Asymptotic expansions for Fuchsian hyperbolic partial differential equations, J. Math. Soc. Japan, 36 (1984), $449-473$.

[5] H. Tahara, Singular hyperbolic systems, VI. Asymptotic analysis for Fuchsian hyperbolic equations in Gevrey classes, J. Math. Soc. Japan, 39 (1987), 551-580.

\section{Department of Mathematics \\ SOPHIA UNIVERSITY \\ KIOICHO, CHIYODA-KU \\ TOKYO 102 \\ JAPAN}

